\title{
First observation of Mars with XMM-Newton
}

\section{High resolution X-ray spectroscopy with RGS}

\author{
K. Dennerl ${ }^{1}$, C. M. Lisse ${ }^{2}$, A. Bhardwaj ${ }^{3}$, V. Burwitz ${ }^{1}$, J. Englhauser ${ }^{1}$, H. Gunell ${ }^{4}$, M. Holmström ${ }^{4}$, F. Jansen ${ }^{5}$, \\ V. Kharchenko ${ }^{6}$, and P. M. Rodríguez-Pascual ${ }^{7}$
}

\author{
1 Max-Planck-Institut für extraterrestrische Physik, Giessenbachstraße, 85748 Garching, Germany \\ e-mail: kod@mpe.mpg.de \\ 2 Johns Hopkins University APL, 11100 Johns Hopkins Road, Laurel, MD 20723, USA \\ 3 NASA Marshall Space Flight Center, NSSTC/XD12, 320 Sparkman Drive, Huntsville, AL 35805, USA \\ On leave from: Space Physics Laboratory, Vikram Sarabhai Space Center, Trivandrum 695022, India \\ 4 Swedish Institute of Space Physics, Box 812, 98128 Kiruna, Sweden \\ 5 European Space Agency, ESTEC Postbus 299, Noordwijk 2200AG, The Netherlands \\ ${ }^{6}$ Harvard-Smithsonian Center for Astrophysics, 60 Garden Street, Cambridge, MA 02138, USA \\ 7 XMM-Newton SOC, Apartado 50727 Villafranca, Madrid 28080, Spain
}

Received 24 September 2005 / Accepted 30 December 2005

\begin{abstract}
In the first observation of Mars with XMM-Newton, on 20-21 November 2003, this planet is clearly detected as an X-ray source. High resolution X-ray spectroscopy with the Reflection Grating Spectrometer (RGS) confirms that the X-ray radiation from Mars is composed of two different components: one due to fluorescent scattering of solar X-rays in its upper atmosphere and the other one due to solar wind charge exchange in its exosphere. Close to Mars, the RGS spectrum is dominated by two pronounced $\mathrm{CO}_{2}$ fluorescence lines at $23.5 \AA$ and $23.7 \AA$. Fluorescence from $N_{2}$ at $31.5 \AA$ is also observed. With increasing distance from Mars, these lines fade, while numerous ( 12) emission lines become prominent at the positions expected for de-excitation of highly ionized $\mathrm{C}, \mathrm{N}, \mathrm{O}$, and $\mathrm{Ne}$ atoms, strongly resembling a cometary $\mathrm{X}$-ray spectrum. The He-like $\mathrm{O}^{6+}$ multiplet is resolved and is dominated by the spin-forbidden magnetic dipole transition $2{ }^{3} \mathrm{~S}_{1} \rightarrow 1^{1} \mathrm{~S}_{0}$, confirming charge exchange as the origin of the emission, while the resonance line $2{ }^{1} \mathrm{P}_{1} \rightarrow 1{ }^{1} \mathrm{~S}_{0}$ increases in intensity closer to Mars, where the density of the exosphere is higher. The high spectral dispersion and throughput of XMM-Newton/RGS make it possible to produce X-ray images of the Martian exosphere in individual emission lines, free from fluorescent radiation. They show extended emission out to $\sim 8 \mathrm{Mars}$ radii, with morphological differences between individual ions and ionization states. This is the first definite detection of charge exchange induced X-ray emission from the exosphere of another planet, providing a direct link to cometary X-ray emission.
\end{abstract}

Key words. atomic processes - molecular processes - solar wind - Sun: X-rays, gamma rays - planets and satellites: individual: Mars X-rays: individuals: Mars

\section{Introduction}

X-rays from Mars were detected for the first time in 2001 with Chandra (Dennerl 2002). The morphology and the X-ray luminosity of $\sim 4 \mathrm{MW}$ were found to be consistent with fluorescent scattering of solar X-rays in the upper Martian atmosphere. The X-ray spectrum obtained with Chandra ACIS-I was dominated by a single narrow emission line at $0.65 \mathrm{keV}$, which was identified as the $\mathrm{O}-\mathrm{K}_{\alpha}$ fluorescence line, shifted by $\sim 120 \mathrm{eV}$ to higher energies due to the additional charge deposited by optical photons in the X-ray CCD ("optical loading"). In addition to the X-ray fluorescence, there was evidence for an additional source of X-ray emission. This was indicated by (i) a faint X-ray halo around Mars which could be traced out to three
Mars radii, and (ii) an additional component in the X-ray spectrum of Mars, which had a similar spectral shape as the halo.

The statistical significance of the Martian halo, however, was quite low, and all the information about it had to be derived from an excess of only $34.6 \pm 8.4(1 \sigma)$ counts relative to the background. Within these very limited statistics, the general spectral properties of this component resembled those expected for charge exchange interactions between highly charged heavy ions in the solar wind and exospheric hydrogen and oxygen around Mars. Recent detailed simulations of this process by Gunell et al. (2004) agreed well with the observed morphology and luminosity of the halo.

Here we report on the first XMM-Newton observation of Mars, performed in November 2003. This observation confirms 
the presence of the halo around Mars and the interpretation above. Spectral studies, now possible in much greater detail, reveal the unambiguous signatures of charge exchange processes and the striking similarity to cometary X-ray spectra. In this paper we concentrate on the results of high resolution X-ray spectroscopy obtained with the Reflection Grating Spectrometers (RGS). The results obtained with the European Photon Imaging Camera (EPIC), which provide a wealth of additional information about the temporal properties of the Martian X-ray radiation due to their high sensitivity, will be presented in a separate paper.

The RGS data also reveal fine structure in the fluorescence spectrum which has not been seen before. This spectrum originates in a shell of $\sim 50 \mathrm{~km}$ thickness in the upper atmosphere centered at $\sim 120 \mathrm{~km}$ above the surface of Mars (Dennerl 2002). Compared to the size of Mars (mean radius: $3389.5 \mathrm{~km}$ ), this shell is very thin and close to the surface. When viewed from a large distance with the spatial resolution of XMM-Newton, the fluorescence radiation appears to originate from the disk of Mars. The situation is quite different for the X-ray emission from the Martian exosphere, which can be traced out to several Mars radii. For easier reference, we will use the terms disk and halo emission for these components in the following sections.

\section{Observation}

\subsection{XMM-Newton and RGS}

XMM-Newton, launched on 10 December 1999, carries three aligned and tightly nested Wolter type I telescopes with a total effective area of more than $0.42 \mathrm{~m}^{2}$ between 0.1 and $2.0 \mathrm{keV}$ (Jansen et al. 2001), providing the highest throughput currently flying. About $50 \%$ of the X-rays entering two of the telescopes reach the Reflection Grating Spectrometers (RGS1 and RGS2, two identical, but independent instruments, operated simultaneously). These provide an energy resolution $E / \Delta E$ between 100 and 600 in the energy range $0.33-2.1 \mathrm{keV}$ (den Herder et al. 2001). Their field of view (FOV) is $5^{\prime}$ along the cross dispersion direction and $\sim 5^{\prime}$ along the dispersion direction. To date, the highest resolution spectrum of an X-ray source in the solar system was obtained with the Chandra/LETG on Venus (Dennerl et al. 2002). Here we present new, higher resolution results on Mars obtained with the RGS instruments.

In the context of observing Mars with RGS, it is important to note that Mars is an extended object and that RGS is a slitless spectrograph. If an extended object emitting only at several distinct wavelengths is observed with a slitless spectrograph, then monochromatic images of the object will appear at the corresponding wavelengths, and the observed spectral lines will exhibit an additional broadening due to the extent of the object. Thus, for an extended object, the signal along the dispersion direction is a superposition of both spectral and spatial properties of the object. If the emission lines are intrinsically broadenend, then the images will become convolved with the line profiles along the dispersion direction (the cross dispersion direction will not be affected). If emission lines are close together in wavelength, then the individual images may overlap and severely degrade the spectral and spatial resolution, unless there is additional information available about the morphology or the spectrum.

RGS is characterized by a high dispersion, which rises monotonically from $3.4 / \AA ̊$ at $35 \AA$ to $4.6 / \AA$ at $10 \AA$. In comparison, the apparent diameter of Mars during the XMM-Newton observation was only 12 '. 2 , and most of the flux from its extended X-ray halo was observed to originate well within $1^{\prime}$ around its center. This fortunate case of observing a moderately extended object emitting only at few distinct wavelengths with a slitless spectrograph of high dispersion and high sensitivity provided a wealth of new information, as the following sections will show.

\subsection{Time of the observation}

As observations with XMM-Newton are restricted to targets at solar elongations between $70^{\circ}$ and $110^{\circ}$, Mars cannot be observed at opposition, when it is closest to Earth. Within the accessible range of solar elongations, values near $110^{\circ}$ are most favorable, because Mars is then considerably closer than at smaller elongations. Due to the eccentricity of the Mars orbit, the actual distance between Mars and Earth at specific elongations is changing with time: the apparent diameter of Mars at $110^{\circ}$ elongation ranges between 8.' 9 and 14 .' 4 .

XMM-Newton was pointed towards Mars from 2003 Nov. 19, 23:47 to Nov. 21, 05:05 UT. During the XMM-Newton observation, Mars had an apparent diameter of 12'.2 Its heliocentric distance was $1.43 \mathrm{AU}$, its geocentric distance 0.77 $\mathrm{AU}$, and it was observed at a phase angle (angle Sun-Mars-Earth) of 41.2 and at a solar elongation of $108^{\circ}$.

\subsection{Moving target}

As seen from XMM-Newton, Mars moved 35'6 with respect to background sources during the observing period, at a rate of $1.2 / \mathrm{h}$. In order to keep Mars well inside the RGS FOV, the satellite was reoriented several times, resulting in 12 periods of stable pointings (Table 1). There was no need for active tracking, because the RGS CCDs were read out every $4.6 \mathrm{~s}$ (implying 0.'09 of apparent motion during a measurement, much less than 1 pixel wide), so that a post-facto transformation of the data into the reference frame of the moving Mars was possible. Compared to the regular XMM-Newton observing mode (stable pointing toward a fixed target), this method of observing had the advantage that any inhomogeneities due to CCD gaps are automatically smoothed and that the spatial resolution is less restricted by the pixel size. The observation was performed in a specific mode, which we had developed for moving targets (Dennerl et al. 2003): the EPIC and RGS instruments were initialized only at the beginning of the observation and stayed on during the whole period (including the times of reorienting the satellite). By avoiding time-consuming recalculations of the offset map, we could considerably increase the observing efficiency.

The fact that the apparent motion of Mars is mainly along the ecliptic plane combined with the roll angle requirements of XMM-Newton implies that Mars moves predominantly along 
Table 1. Journal of stable pointings ${ }^{\star}$ of XMM-Newton, with RGS in observing mode.

\begin{tabular}{rcccccc}
\hline \hline \multicolumn{1}{c}{ Pnt } & \multicolumn{2}{c}{ Begin } & \multicolumn{2}{c}{ End } & Duration & Roll \\
$\#$ & 2003 & {$[\mathrm{UTC}]$} & 2003 & {$[\mathrm{UTC}]$} & \multicolumn{1}{c}{$[\mathrm{s}]$} & {$[\mathrm{deg}]$} \\
\hline 1 & Nov. 19 & $23: 48: 58$ & Nov. 20 & $02: 43: 40$ & 10482 & 232.8 \\
2 & Nov. 20 & $02: 55: 22$ & Nov. 20 & $05: 16: 01$ & 8438 & 234.8 \\
3 & Nov. 20 & $05: 29: 05$ & Nov. 20 & $07: 45: 20$ & 8175 & 236.8 \\
4 & Nov. 20 & $07: 57: 03$ & Nov. 20 & $10: 18: 40$ & 8497 & 238.8 \\
5 & Nov. 20 & $10: 28: 23$ & Nov. 20 & $12: 42: 00$ & 8017 & 240.8 \\
6 & Nov. 20 & $12: 49: 56$ & Nov. 20 & $15: 03: 40$ & 8024 & 241.8 \\
7 & Nov. 20 & $15: 11: 45$ & Nov. 20 & $17: 23: 40$ & 7915 & 242.8 \\
8 & Nov. 20 & $17: 33: 23$ & Nov. 20 & $19: 47: 00$ & 8017 & 243.8 \\
9 & Nov. 20 & $19: 56: 43$ & Nov. 20 & $22: 08: 40$ & 7917 & 244.8 \\
10 & Nov. 20 & $22: 18: 33$ & Nov. 21 & $00: 29: 17$ & 7844 & 245.8 \\
11 & Nov. 21 & $00: 38: 13$ & Nov. 21 & $02: 48: 40$ & 7827 & 246.8 \\
12 & Nov. 21 & $02: 58: 13$ & Nov. 21 & $04: 58: 53$ & 7240 & 247.8 \\
\hline
\end{tabular}

* Defined by the time intervals with less than 1 " in spacecraft jitter. Roll angle: angle between equatorial north and decreasing dispersion direction, measured counterclockwise.

the RGS dispersion direction. The parallax due to the orbit of XMM-Newton, however, introduces a curvature in the apparent path of Mars. In order to keep Mars at similar cross-dispersion distances, the roll angle of the satellite was adjusted for each individual pointing so that Mars would move mainly along the RGS dispersion direction (Table 1). As a compromise to the requirements of EPIC pn, we shifted the track of Mars by 0.' 6 along the cross dispersion direction away from the nominal aimpoint $^{1}$.

\subsection{Optical light}

One important property of RGS in the context of the Mars observation is that the optical flux is completely separated from the X-ray flux by diffraction. Thus, the high optical surface brightness of Mars (+4.4 mag $\operatorname{arcsec}^{-2}$ at the time of the observation), which is a major point of concern for many current $\mathrm{X}$-ray detectors (e.g. Chandra ACIS-S), is no problem for RGS and does not affect the RGS spectra in any way.

\section{Data analysis}

The specific properties of this observation required several nonstandard methods of data reduction, which are described below.

\subsection{Stable pointing periods}

Due to the special observing mode applied (Sect. 2.3), it was necessary to identify first the 12 time intervals of stable pointing (when the spacecraft jitter was smaller than $1^{\prime \prime}$; Table 1) and to suppress the data which were taken in between. This ensured that the reconstructed image and spectrum of Mars would not be affected by any blur which might result from increased uncertainties about the satellite attitude during the intervals when XMM-Newton was reoriented.

1 This increased the distance between Mars and the gap between the EPIC pn CCDs 4 and 7 and minimized the risk that part of the X-ray halo might fall into this gap.

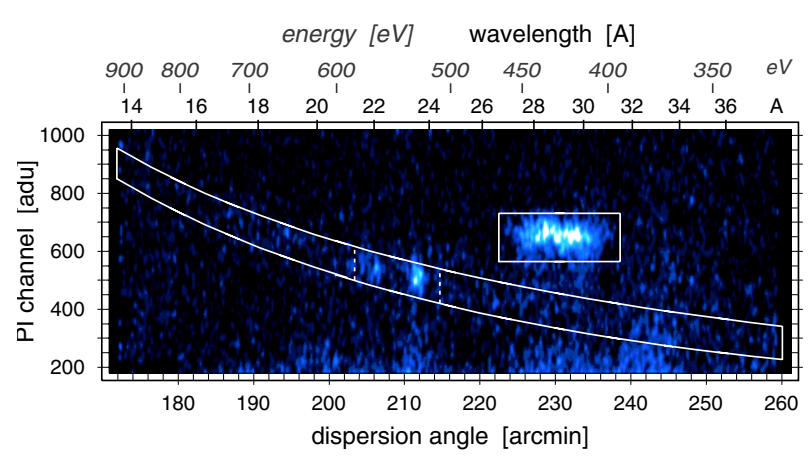

Fig. 1. Intrinsic energy resolution of RGS 1 as a function of the dispersion angle, accumulated during a total of $74 \mathrm{ks}$ of stable pointing and low instrumental background, and transformed into the rest frame of Mars. This image was corrected for exposure variations, was binned into $10^{\prime \prime}$ dispersion angle $\times 5$ adu pixels and smoothed with a Gaussian function with $\sigma_{x}=10^{\prime \prime}$ and $\sigma_{y}=10 \mathrm{adu}$; the color scale extends linearly from zero (black) to $3.3 \times 10^{-5}$ counts $\operatorname{arcmin}^{-1} \mathrm{adu}^{-1} \mathrm{~s}^{-1}$ (white). Only events within the curved region were used for the spectral studies (see text). A spectral image of the region between the dashed lines is shown in Fig. 5b. Events inside the horizontal rectangle were caused by an internal calibration source.

\subsection{Particle background}

The observation was characterized by episodes of high particle background. However, these background flares dominate at high energies, while the spectral range of interest for this analysis is confined to energies below $\sim 1 \mathrm{keV}$. Furthermore, any background in the RGS CCDs can be suppressed efficiently by using the CCD intrinsic energy resolution to screen photons by their pulse height spectra (i.e., by demanding that photons as a given dispersion angle also have the corresponding CCD pulse height energy; Fig. 1). Thus, even episodes of moderately increased particle background did not degrade the data quality substantially. The whole data set $(98 \mathrm{ks}$, Table 1) could be used for spectral studies of the immediate environment of Mars, at cross dispersion distances $|y| \leq 10^{\prime \prime}$. For studies of the extended halo emission, the requirements for a clean signal are higher, and we used only periods of low background (less than 0.3 counts $\mathrm{s}^{-1}$ in CCD 5 of RGS 1 ), which yielded a total low background time of $74 \mathrm{ks}$.

\subsection{Transformation into the rest frame of Mars}

Transformation of the photons and the exposure into the moving reference frame of Mars was done using the geocentric Mars ephemeris from $\mathrm{JPL}^{2}$ and the information about the XMM-Newton attitude and orbit contained in the delivered data set. We transformed them into a reference system where the positive $x$ axis is the direction of increasing dispersion angle, which is approximately antiparallel to the direction of the Sun. The same transformation was used for creating the corresponding exposure maps. As a consequence of the changing roll angle of the satellite (Sect. 2.3), the angle between the Sun with respect to the direction of decreasing dispersion was slowly varying from pointing to pointing, from -13.9 to $+1^{\circ} .1$.

\footnotetext{
2 Available at http://ssd.jpl.nasa.gov/cgi-bin/eph
} 
The fact that RGS is a diffractive spectrograph also requires to consider the velocity of Mars with respect to XMM-Newton along the line of sight in the transformation to the Martian reference frame, because this velocity introduces a shift along the dispersion direction. However, the radial velocity of Mars with respect to XMM-Newton was only between +13.5 and $+14.2 \mathrm{~km} \mathrm{~s}^{-1}$ (with $+13.2 \mathrm{~km} \mathrm{~s}^{-1}$ coming from the radial velocity between Mars and Earth), causing a negligible shift of less than +0.35 in the dispersion direction. The situation is different for the solar wind ions, which are ejected from the Sun with velocities of $400-800 \mathrm{~km} \mathrm{~s}^{-1}$. Their interaction with the Martian exosphere (by charge exchange) might lead to a detectable redshift in the emission. In the transformation to the Martian reference frame this shift cannot be taken into account, because the specific velocities are not known precisely enough. The presence and potential consequences of such redshifts will be investigated in more detail in Sect. 5.1.

\subsection{Unrelated X-ray sources}

Like any object moving across the sky, Mars passed unrelated $\mathrm{X}$-ray sources, which could potentially contaminate its X-ray signal. For a direct imaging device like EPIC, the contamination of the signal can be avoided in a straightforward way, by ignoring the photons from and around such sources. For a slitless diffracting device like RGS, however, the situation is different due to the superposition of spatial and spectral information along the dispersion direction. Depending on the spectrum, photons from a single source may be distributed over a wide range in dispersion direction. As Mars was moving predominantly along this direction, the most conservative way of removing all photons within the diffracted spectra of unrelated $\mathrm{X}$-ray sources (identified with the simultaneous EPIC data) would also remove most of the photons from Mars, even if the intrinsic energy resolution of the RGS CCDs (Fig. 1) were used for minimizing the amount of data to be removed.

However, in the case of this specific observation, it is possible to demonstrate that contamination of the Martian spectrum by unrelated X-ray sources is negligible, because this spectrum is found to be dominated by few narrow emission lines. Transformed into the reference system of Mars, spectra of background sources along its path would not show such narrow features, because of their apparent drift along the dispersion direction. Moreover, the X-ray spectra of many background sources are characterized by continuum radiation. In particular, this is the case for the brightest X-ray source along the path of $\mathrm{Mars}^{3}$, as seen in the EPIC data.

\subsection{X-ray background and detector noise}

As the halo of Mars is a very faint X-ray source for RGS, an efficient background suppression is essential in order to get a clean signal. For RGS, this is possible by utilizing the fact that the dispersed spectrum is measured with a detector which has

\footnotetext{
${ }^{3}$ This is a previously undetected X-ray source with a powerlaw spectrum, probably an AGN, coincident with the $v_{b}=17.9$ mag object U0825_19872482 of the USNO-A2.0 Catalogue.
}

an intrinsic energy resolution. By combining the information about the pulse height energy from this detector with the dispersion information, the signal to noise ratio can be considerably enhanced (see Sect. 3.2).

Compared to the standard procedure for event selection, we applied more stringent limits to the pulse heights as a function of the dispersion angle computed for the rest frame of Mars, in order to improve the signal to noise ratio (Fig. 1). For all spectral fits, the background was determined from two bands above and below Mars along the dispersion direction, from cross dispersions $|y| \geq 1.3$ to the borders of the CCDs (e.g. Fig. 5b), after applying the same pulse height selection.

\subsection{Spatial/spectral deconvolution}

The PSF (point spread function) of the XMM-Newton telescopes is characterized by a FWHM (full width at half maximum) of $\sim 5^{\prime \prime}$. For RGS, the effective $F W H M$ is somewhat larger due to the convolution with the grating response and the finite pixel size $\left(2.5^{\prime \prime} \times 2.5^{\prime \prime}\right)$, and is comparable to the apparent size of Mars (12'.2) during the observation. Thus, Mars is only marginally resolved, and some X-rays from its upper atmosphere are spread by the PSF into the halo, while the X-rays which we see from the disk of Mars contain some contribution from the halo. On the other hand, as both components emit at different wavelengths/energies, the high spectral dispersion of the RGS $\left(\sim 4^{\prime} / \AA\right)$ makes it possible to clearly separate the radiation from the disk and halo.

\section{Observational results}

\subsection{The total spectrum of Mars and its halo}

The RGS spectrum of Mars and its halo, accumulated at cross dispersion distances $|y| \leq 50^{\prime \prime}$ from the center of Mars (Fig. 2) reveals the presence of many emission lines. These lines can be identified with either fluorescence of neutral molecules ("disk lines") or with de-excitation of highly ionized atoms ("halo lines"), as Table 2 shows. Because the statistical quality of some emission lines is too low for an individual determination of their widths, we proceeded as follows. We grouped the emission lines into two classes, according to their probable origin: disk or halo. The widths of all lines for each class were fixed in $\AA$ to the same value, in order to allow for different spatial extents in both groups. The positions (energy/wavelength) and intensities of all lines were free parameters in the fit.

The resulting best-fit model spectrum is shown in Fig. 2, and the spectral parameters are listed in Table 2a. We find in all cases an excellent agreement between the observed line energies and those expected for characteristic electron transitions in $\mathrm{C}, \mathrm{N}, \mathrm{O}$ atoms, as listed in Table $2 \mathrm{~b}$. All these are transitions into the ground state. For easier reference, we use in the following the abbreviations listed in Table $2 \mathrm{~b}$. These abbreviations are generally composed of the atom name followed by its ionization state and the shell number from which the electron transition starts. We have additionally assigned running numbers to the emission lines according to increasing wavelength and use them consistently throughout the paper. 


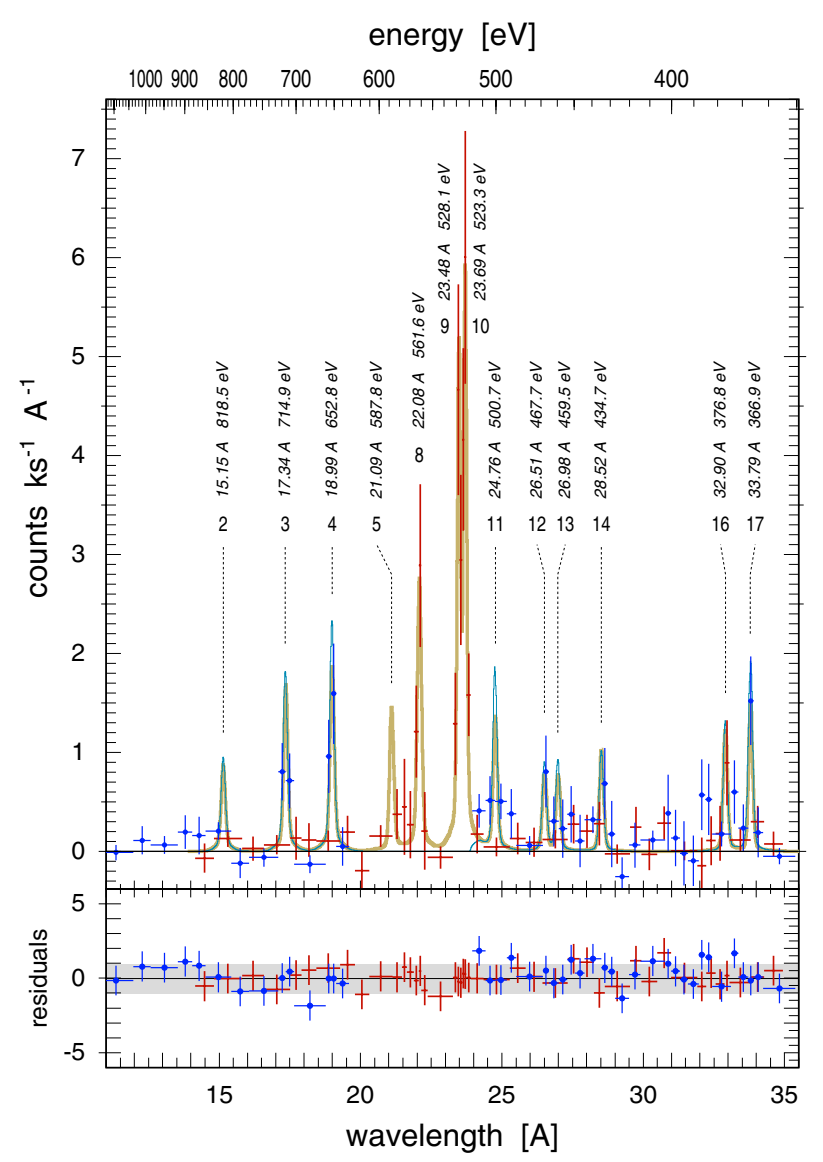

Fig. 2. RGS spectra of Mars and its halo, accumulated at cross dispersion distances $|y| \leq 50^{\prime \prime}$ from the center of Mars during $74.1 \mathrm{ks}$ (excluding the times of high instrumental background). The RGS data were binned to contain at least 25 events and are plotted as crosses with $1 \sigma$ error bars in intensity; RGS 2 data are marked with an additional dot at the center. For parts of the spectrum, data exist from only one instrument. The data from both spectrometers were fit together, using their individual response matrices. Smooth curves show the corresponding folded model spectra, with a thick line for RGS 1 and a thin one for RGS 2. The positions resulting from the fit (in $\AA$ and eV) are written above each line, together with a running number, for easier reference. Parameters obtained from this fit are listed in Table 2a. A zoomed version of the central region around $23 \AA / 540 \mathrm{eV}$ is shown in Fig. 5.

Table $2 \mathrm{~b}$ contains also some emission lines which we find only in the disk or halo spectrum (see below), due to an improved signal to noise ratio in these restricted extraction regions. Because of the importance of the He-like $\mathrm{O}^{6+}$ multiplet (cf. Sect. 5.3), we also list in Table $2 \mathrm{~b}$ the intercombination line O6i (\#7), although it is not detected. For completeness, we include the emission lines \#2 and \#5 in Table 2, which are indicated in the data, but are rather uncertain. The 17 emission lines listed in this table represent the basis for the subsequent analysis. Confident in our line detections and instrument characterization, we fixed in the next step the line wavelengths/energies to these values, allowing for a tolerance of $\pm 0.1 \AA$ (or $\pm \sim 20^{\prime \prime}$ ). This tolerance would take systematic shifts into account that

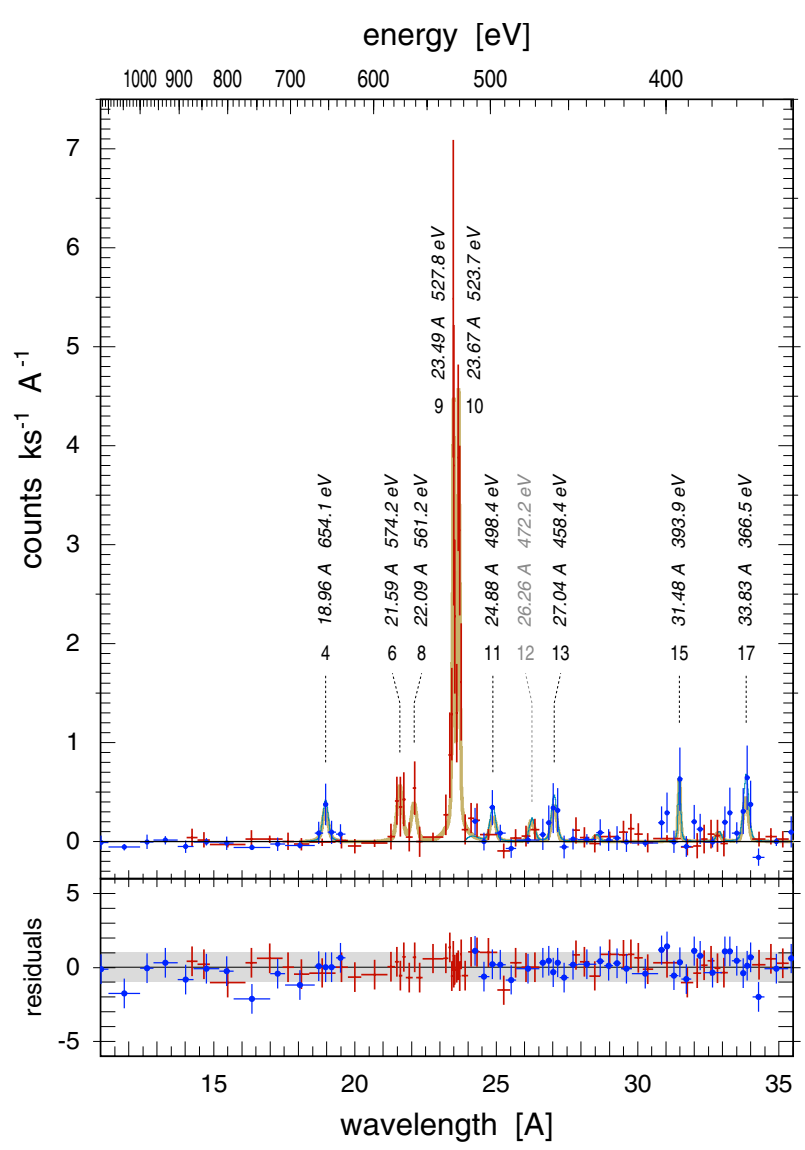

Fig. 3. Disk spectrum: same as Fig. 2, but for cross dispersion distances $|y| \leq 10^{\prime \prime}$ from the center of Mars. The positions of all lines were constrained to $\pm 0.1 \AA$ of the expected values (Table 2b). A common width (in $\AA$ ) was determined for the lines \#9 and \#10, as well as for the other lines. The fit gives a reduced $\chi_{v}^{2}=0.69$ for 78 degrees of freedom. Fluxes derived from this fit are listed in Table $2 \mathrm{c}$.

might result from to the fact that these lines do not originate exactly at the position of Mars.

Within the He-like $\mathrm{O}^{6+}$ multiplet, the positions of lines O6r and O6i were fixed relative to O6f, according to Table $2 \mathrm{~b}$. The widths of the fluorescence lines CO2a, CO2b, and N22 were fixed to the same, adjustable value (in $\AA$ ), and the widths of the remaining lines were coupled in a similar way.

\subsection{The disk spectrum}

In Fig. 3 we show RGS spectra of Mars at cross dispersion distances $|y| \leq 10^{\prime \prime}$ from its center, accumulated during $98.0 \mathrm{ks}$ (including times of high instrumental background, but excluding the intervals when the satellite was reoriented). The RGS data were binned to contain at least 10 events, in order to minimize loss of spectral resolution; errors were determined with the $C$ statistics (Cash 1979). This extraction region is dominated by emission from close to Mars along the cross dispersion direction, but may also contain contributions from outer areas along the dispersion direction.

The main difference between the disk spectrum (Fig. 3) and the total (disk+halo) spectrum (Fig. 2) is that in the disk spectrum most emission lines are considerably fainter, except lines \#9 and \#10. These two lines are also sharper: by fitting 
Table 2. Emission lines in the RGS spectra of Mars and its halo.

\begin{tabular}{|c|c|c|c|c|c|c|c|c|c|c|c|}
\hline \multicolumn{2}{|c|}{ Observed line position } & \multirow{2}{*}{$\begin{array}{c}\text { Photon flux } \\
{\left[10^{-6} \mathrm{~cm}^{-2} \mathrm{~s}^{-1}\right]}\end{array}$} & \multicolumn{2}{|c|}{ Line id } & \multicolumn{2}{|c|}{ Line origin } & \multirow{2}{*}{$\begin{array}{c}\lambda \\
{[\AA]}\end{array}$} & \multirow{2}{*}{$\begin{array}{c}E \\
{[\mathrm{eV}]}\end{array}$} & \multicolumn{3}{|c|}{ Photon flux $\left[10^{-6} \mathrm{~cm}^{-2} \mathrm{~s}^{-1}\right]$} \\
\hline \multirow[t]{2}{*}{$\lambda[\AA]$} & \multirow[t]{2}{*}{$E[\mathrm{eV}]$} & & $\#$ & Abbr & Ion & Transition & & & Total & Disk region & Halo region \\
\hline & & & 1 & $\mathrm{Ne} 72$ & $\mathrm{Ne}^{7+}$ & $2 p \rightarrow 1 s$ & 14.21 & 872.5 & $(1.8 \pm 1.8)$ & $(0.5 \pm 0.5)$ & $3.8 \pm 2.2$ \\
\hline $15.15 \pm 0.10$ & $818.5 \pm 5.5$ & 3.9 & 2 & $\mathrm{O} 74$ & $\mathrm{O}^{7+}$ & $4 p \rightarrow 1 s$ & 15.18 & 817.0 & $3.9 \pm$ & ) & $.6)$ \\
\hline & 714.9 & & 3 & O65 & $\mathrm{O}^{6+}$ & $5 p \rightarrow 1 s$ & 7.40 & 712.5 & $7.9 \pm 2.1$ & $(0.4 \pm 0.4)$ & \\
\hline $18.99 \pm$ & $652.8 \pm 1.0$ & $0 \quad 9.9=$ & 4 & $\mathrm{O} 72$ & $\mathrm{O}^{7+}$ & $2 p \rightarrow 1 s$ & 18.97 & 653.6 & $9.9 \pm 2.4$ & $4.3 \pm 2.1$ & $7.4 \pm 2.1$ \\
\hline \multirow[t]{3}{*}{$21.09 \pm 0.07$} & \multirow[t]{3}{*}{$587.8 \pm 1.8$} & \multirow[t]{3}{*}{$7.5 \pm 3.9$} & 5 & N63 & $\mathrm{N}^{6+}$ & $3 p \rightarrow 1 s$ & 91 & 0 & $38.1 \pm 30$ & $(2.4 \pm 2.4)$ & $22.0 \pm 19$ \\
\hline & & & 6 & O6r & $\mathrm{O}^{6+}$ & ${ }^{1} \mathrm{P}_{1} \rightarrow{ }^{1} \mathrm{~S}_{0}$ & 21.60 & & & & \\
\hline & & & 7 & O6i & $\mathrm{O}^{6+}$ & $2{ }^{3} \mathrm{P}_{1} \rightarrow 1^{1} \mathrm{~S}_{0}$ & 21.81 & 568.5 & $(1.5 \pm$ & (1.2 & $(2.2=$ \\
\hline $22.08 \pm 0.02$ & $561.6 \pm 0.6$ & $\begin{array}{ll}6 & 13.5 \\
\end{array}$ & 8 & O6f & $\mathrm{O}^{6+}$ & ${ }^{3} \mathrm{~S}_{1} \rightarrow 1^{1} \mathrm{~S}_{0}$ & 22.11 & 560.9 & $12.8 \pm 3.2$ & $4.2 \pm 2.9$ & 2.3 \\
\hline $23.48 \pm 0.02$ & $528.1 \pm 0.4$ & $419.8 \pm 3.8$ & 9 & $\mathrm{CO} 2 \mathrm{a}$ & $\mathrm{CO}_{2}$ & $1 \pi_{g} \rightarrow 1 \mathrm{~s}$ & 23.50 & 527.7 & $5+37$ & 23 & .8 \\
\hline $23.69 \pm 0.01$ & $523.3 \pm 0.3$ & $323.1 \pm 3.7$ & 10 & $\mathrm{CO} 2 \mathrm{~b}$ & $\mathrm{CO}_{2}$ & $3 \sigma_{u} \rightarrow 1 \mathrm{~s}$ & 23.68 & 523.5 & $23.6 \pm 3.5$ & $21.7 \pm 3.8$ & $3.6 \pm 2.8$ \\
\hline $24.76 \pm 0.03$ & $500.7 \pm 0.7$ & $7 \quad 9.1 \pm 2.8$ & 11 & N62 & $\mathrm{N}^{6+}$ & $2 p \rightarrow 1 s$ & 24.78 & 500.3 & $9.3 \pm 2.7$ & $4.5 \pm 2.2$ & $7.1 \pm 2.2$ \\
\hline 26.5 & 467. & 1 & 12 & $\mathrm{C} 55$ & $\mathrm{C}^{5+}$ & $5 p-$ & 26.36 & 470 & $5.5 \pm$ & 2.7 & 5.5 \\
\hline $26.98 \pm 0.08$ & 459.5 & $4 \quad 4.2$ & 13 & $\mathrm{C} 54$ & $\mathrm{C}^{5+}$ & $4 p \rightarrow 1 s$ & 26.99 & 459.4 & $4.5 \pm 3.2$ & $5.7 \pm 2.8$ & $5.3 \pm 2.5$ \\
\hline \multirow[t]{2}{*}{$28.52 \pm 0.06$} & $434.7 \pm 1.0$ & $6.3 \pm 2.5$ & 14 & C53 & $\mathrm{C}^{5+}$ & $3 p \rightarrow 1 s$ & 28.47 & 435.6 & $6.6 \pm 2.3$ & $(1.9 \pm 1.9)$ & $7.9 \pm 2.7$ \\
\hline & & & 15 & $\mathrm{~N} 22$ & $\mathrm{~N}_{2}$ & $3 \sigma_{g} \rightarrow 1 \mathrm{~s}$ & 31.47 & 394.0 & $(2.2 \pm 2.2)$ & $5.8 \pm 2.8$ & $(1.4 \pm 1.4)$ \\
\hline 32.90 & 376. & & 16 & $\mathrm{C} 45$ & $\mathrm{C}^{4+}$ & $5 p \rightarrow 1 s$ & 32.75 & 37 & $9.9 \pm 4.2$ & $(2$. & \\
\hline $33.79 \pm 0.04$ & $366.9 \pm 0.5$ & $514.8 \pm 4.5$ & 17 & C52 & $\mathrm{C}^{5+}$ & $2 p \rightarrow 1 s$ & 33.74 & 367.6 & $16.2 \pm 5.2$ & $11.0 \pm 4.2$ & $9.1 \pm 3.5$ \\
\hline
\end{tabular}

a) emission lines found at $|y| \leq 50^{\prime \prime}$

b) line identifications

c) flux derived for fixed line positions

a) Emission lines found at cross dispersion distances $|y| \leq 50^{\prime \prime}$ from the center of Mars (cf. Fig. 2). The positions and fluxes of all lines were treated as free parameters in the fit and are listed with their $1 \sigma$ errors. A common value (in $\AA$ ) was determined for the width of the lines $\mathrm{CO} 2 \mathrm{a}$ and $\mathrm{CO} 2 \mathrm{~b}$, as well as for all the other lines. b) Identification of the emission lines, including lines which are found only in the disk or halo spectrum, plus the intercombination line O6i of the He-like $\mathrm{O}^{6+}$ multiplet. For CO2b, also the transitions $4 \sigma_{q} \rightarrow 1 \mathrm{~s}$ and $1 \pi_{u} \rightarrow 1 \mathrm{~s}$ may contribute to the flux. c) The fluxes are given together with their $1 \sigma$ errors, as determined by the interval where the $C$ value (Cash 1979) of the fit increases by 1.0 , allowing adjustment of all free parameters; the $\Delta \chi^{2}=+1.0$ approach gives similar results. Brackets mark lines of low significance where the $1 \sigma$ error interval includes zero.

common values (in $\AA$ ) to the width of these lines and, independently, to the width of the other lines, we find a $F W H M$ of $(0.05 \pm 0.05) \AA$ for lines \#9 and \#10, but $(0.22 \pm 0.07) \AA$ for the other lines. This indicates that the emission which we see from Mars comes from different regions. Also, two new lines (\#6 and \#15) show up in the disk spectrum, obviously originating close to Mars. The derived photon fluxes for the lines are listed in Table 2c.

\subsection{The halo spectrum}

In order to investigate the properties of the outer regions around Mars, we accumulated RGS spectra at cross dispersion distances $15^{\prime \prime}<|y|<50^{\prime \prime}$ from the center of Mars during $74.1 \mathrm{ks}$ (excluding the times of high instrumental background, see Sect. 3.2). The RGS data were binned to contain at least 20 events. This halo spectrum, displayed in Fig. 4, is considerably different from the disk spectrum (Fig. 3): it exhibits a wealth of emission lines of similar flux, while the lines \#9 and \#10, which dominated the disk spectrum, have almost disappeared.

By fitting a common value (in $\AA$ ) to the width of all lines except \#9 and 10, we obtain a FWHM of $(0.20 \pm 0.11) \AA$, which is similar to that derived for the disk spectrum outside the strong fluorescence lines. We determined the errors with both the $\chi^{2}$ statistics (Lampton et al. 1976) and the $C$ statistics

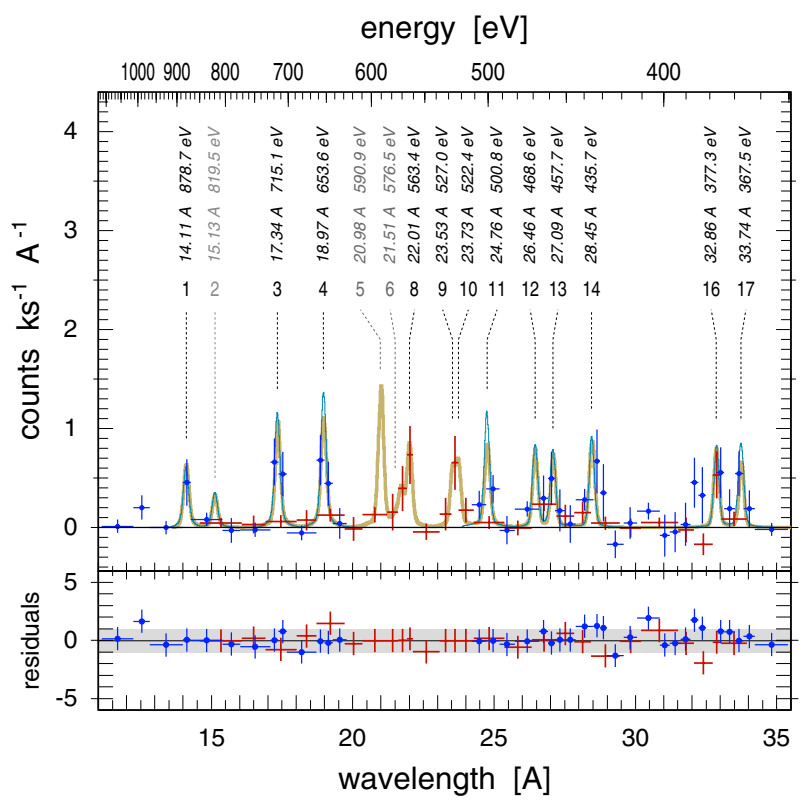

Fig. 4. Halo spectrum: same as Fig. 2, but for cross dispersion distances $15^{\prime \prime}<|y|<50^{\prime \prime}$ from the center of Mars. The positions of all lines were constrained to $\pm 0.1 \AA$ of the expected values (Table $2 b$ ). A common width (in $\AA$ ) was determined for the lines \#9 and \#10, as well as for the other lines. The fit gives a reduced $\chi_{v}^{2}=1.09$ for 30 degrees of freedom. Fluxes derived from this fit are listed in Table $2 \mathrm{c}$. 

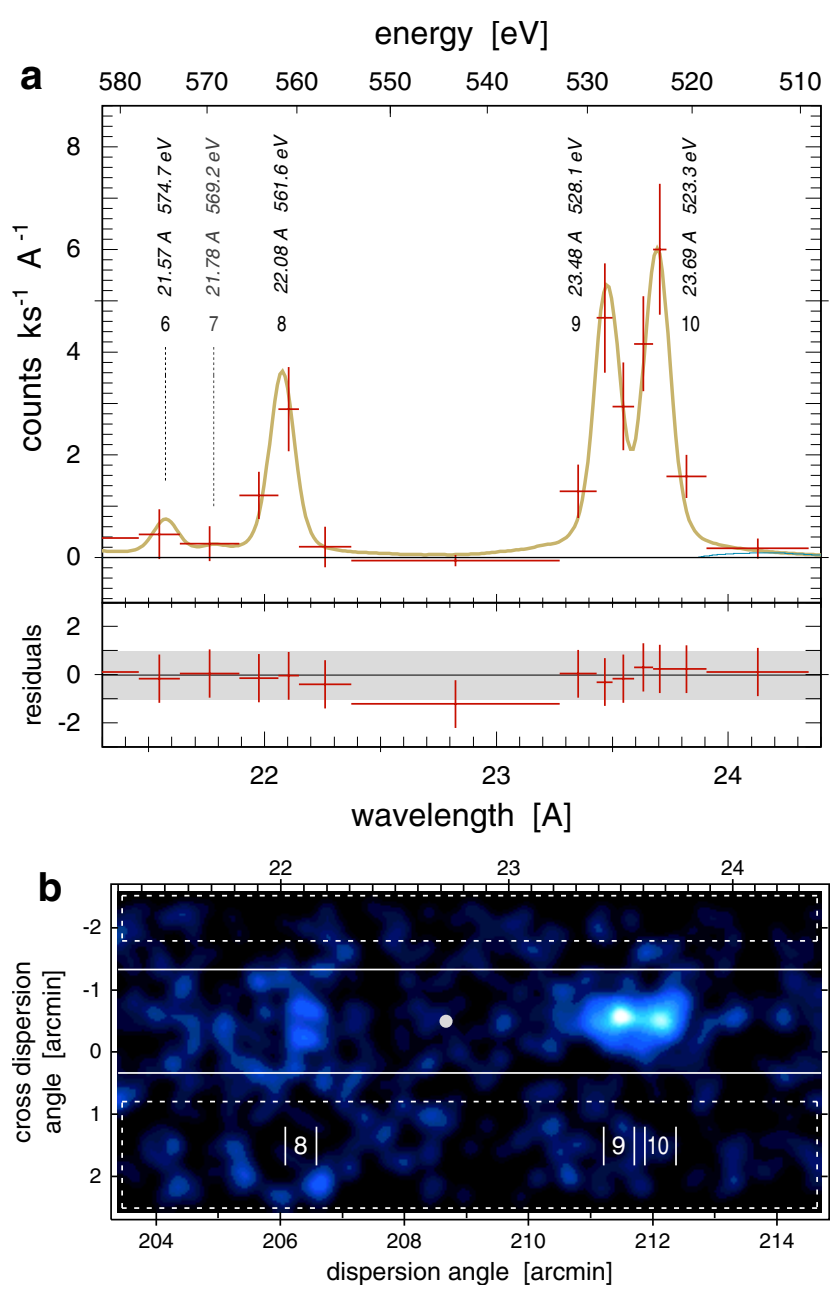

Fig. 5. RGS 1 data from Mars, accumulated during a total of $74 \mathrm{ks}$ of stable pointing and low instrumental background. a) RGS 1 spectrum of Mars and its halo at cross dispersion distances $|y| \leq 50^{\prime \prime}$ from the center of Mars, showing the region around the $\mathrm{CO}_{2}$ doublet and the $\mathrm{O}^{6+}$ multiplet (this region is not covered by RGS 2). b) Dispersed image in the same wavelength/energy range as in a), accumulated from the events inside the curved region in Fig. 1. This image was binned into $2^{\prime \prime} \times 2^{\prime \prime}$ pixels and smoothed with a Gaussian function with $\sigma=8^{\prime \prime} \times 8^{\prime \prime}$; the color scale extends from zero (black) to $2.7 \times 10^{-3}$ counts arcmin ${ }^{-2} \mathrm{~s}^{-1}$ (white). It contains the full FOV of RGS along the cross dispersion direction. The inner rectangle shows the extraction region for the $|y| \leq 50^{\prime \prime}$ spectra; the background was taken from the two dashed bands above and below. The dot at center illustrates the angular size of Mars during the observation; the observed images are blurred mainly due to the PSF of the telescope. The regions for extracting profiles along cross dispersion direction (cf. Fig. 6) are denoted with 8,9, and 10; they cover 30" along the dispersion direction. Note that due to the high dispersion of RGS, the X-ray image of the Mars halo (8) is completely detached from the fluorescent images $(9,10)$.

(Cash 1979) and found consistent results. Since these lines are intrinsically sharp and since any instrumental broadening has been taken into account in the fitting procedure, the width of the lines indicates a spatial extent of the emission region along dispersion direction, with a $F W H M$ of $\sim 45^{\prime \prime}$. The photon fluxes are listed in Table 2c.

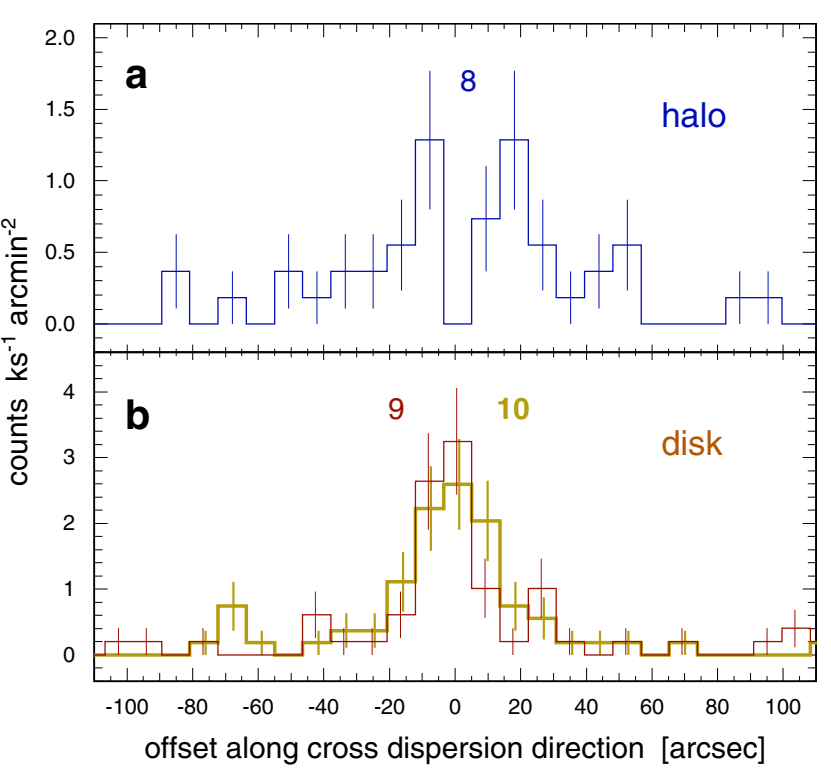

Fig. 6. Brightness profiles (with $1 \sigma$ error bars) along cross dispersion direction of the Martian exosphere (derived from the unsmoothed data) in the light of a) the $2{ }^{3} \mathrm{~S}_{1} \rightarrow{ }^{1} \mathrm{~S}_{0}$ transition of $\mathrm{O}^{6+}$ (\#8) and b) the $\mathrm{CO}_{2}$ fluorescence doublet (\#9, \#10). The extraction geometry is illustrated in Fig. 5b. While the $\mathrm{CO}_{2}$ profiles are very similar, the $\mathrm{O}^{6+}$ profile has a quite different shape, characterized by a pronounced dip at the center.

\subsection{Mars at $21-24 \AA$}

The spectral region around $21-24 \AA(\sim 510-580 \mathrm{eV})$ is particularly interesting: it not only contains the brightest emission lines covered by RGS, but also the diagnostically very important He-like multiplet of $\mathrm{O}^{6+}$. Operating as a slitless spectrograph, the RGS data contain also spatial information, in particular for extended, but sufficiently compact sources, which emit at specific, sharp energies, like Mars. Figure 5 focuses on this spectral region, showing at top the spectrum and at bottom, at the same horizontal scale, the corresponding spectral image.

All the emission in this spectral region originates from inner shell electron transitions in oxygen, either six-fold ionized or neutral. In both cases the RGS spectra and images reveal a wealth of detail:

- The $\mathrm{CO}_{2}$ emission (lines \#9 and \#10) is clearly resolved into two components of similar flux. Both components originate close to the planet, as the spectral image shows: their brightness distributions peak at the position of Mars. Brightness profiles along the cross dispersion direction (Fig. 6b) exhibit no significant difference between both components.

- The He-like $\mathrm{O}^{6+}$ multiplet (lines \#6-8) is also resolved. Compared to Fig. 2 and Table 2a, all components of the "triplet" were included in this fit; their relative positions were fixed and their widths (in $\AA$ ) were set to the same (adjustable) value. The fit shows some indication for the presence of the resonance line O6r (\#6), though not at a significant level; it does not show any evidence for the intercombination line O6i (\#7). The spin-forbidden magnetic dipole transition O6f (\#8), however, is clearly dominating. 
This is a direct proof that charge exchange is the source of the emission (Sect. 5.3).

- There are indications that the flux ratios in the He-like $\mathrm{O}^{6+}$ multiplet change with distance from Mars: the resonance line O6r (\#6), which is almost undetectable in the halo spectrum (Fig. 4), is clearly present in the disk spectrum (Fig. 3), while the flux of the O6f line (\#8) is similar in both spectra (see also Table 2c).

- The spectral image of the O6f transition is completely different from that of the $\mathrm{CO}_{2}$ emission: it shows two distinct blobs along the cross dispersion direction (which is approximately the North-South direction on Mars), with practically no emission in between (Fig. 6a). This means that the emission does not originate close to Mars (the size of Mars is drawn to scale at the center of Fig. $5 b$ for reference) or in an X-ray luminous extended shell around Mars, but at two well localized regions $\sim 3000 \mathrm{~km}$ above both poles.

\subsection{RGS images of Mars}

As demonstrated in the last section, RGS images contain a wealth of information about the morphology of the X-ray emission at specific wavelengths/energies. In Fig. 7, we present images for the major emission lines O72, O6f, C53, C52, CO2a, $\mathrm{CO} 2 \mathrm{a}$, and N22, adding data from RGS 1 and RGS 2 together, whenever available. These images reveal different morphologies, not only between fluorescence and charge exchange X-ray emission, but also between different charge exchange lines.

The structure seen in the spectral image of O6f seems to be a specific property of emission from ionized oxygen, as the O72 image (Fig. 7a) also shows two distinct blobs along cross dispersion direction. Compared to O6f (Fig. 7b), the O72 emission occurs at larger distances from Mars. There is also some evidence in Fig. 7a that the peak of the 072 emission is shifted to the right with respect to Mars. Interpreted as redshift, this would indicate velocities of $\mathrm{O}^{8+}$ ions of $\gtrsim 400 \mathrm{~km} \mathrm{~s}^{-1}$ along the line of sight, as the dashed vertical lines in Fig. 7a indicate. Alternatively, this shift may be interpreted as a spatial displacement. These different scenarios are investigated in more detail in Sect. 5.1.

Spectral images can also be obtained for the carbon emission lines C53 and C52 (Figs. 7d,e). These images show yet another morphology: there is again clear evidence for extended, unisotropic emission, but unlike the blobby $\mathrm{O} 72$ and O6f appearance, the C53 and C52 emissions exhibit a more band-like structure without a pronounced intensity dip at the position of Mars. There is also evidence that at larger distances from Mars the emission is shifted towards the right.

For completeness we show in Fig. $7 \mathrm{~h}$ also the image of the $\mathrm{N} 22$ emission, which demonstrates that, similar to $\mathrm{CO}_{2}$ (Fig. 7g), this radiation is coming from close to Mars, as expected for fluorescence. In Fig. 7i this image has been combined with the two $\mathrm{CO}_{2}$ images, in order to obtain a representative image of Mars in the light of fluorescent radiation. Similarly, the O72 and O6f images, as well as the C53 and C52 images have been added to show the Martian halo in the light of ionized oxygen and carbon (Figs. 7c, f). These stacked images were then combined into a color image, by coding fluorescent radiation in red/yellow, and ionized carbon and oxygen in green and blue, respectively, to produce Fig. 8a. This image will be discussed in the next section.

\section{Discussion}

\subsection{Combined spectral image}

Figure $8 \mathrm{a}$ is a superposition of RGS images of Mars in seven emission lines between 19 and $34 \AA(360-660 \mathrm{eV})$, with ionized oxygen coded in blue, ionized carbon coded in green, and fluorescence coded in yellow and red. The horizontal axis is the dispersion direction, with wavelength increasing to the right. Along this direction, the angular and linear scale at lower right is valid only if the individual images are purely monochromatic and exhibit no wavelength/energy shift. Alternatively, the velocity scale at upper right illustrates the amount of horizontal displacement which would be caused by the Doppler shift. As the wavelength is not a linear function of dispersion in RGS, the amount of displacement depends on the wavelength/energy. The two bars enclose the spectral range used, with the upper (shorter) bar referring to the $\mathrm{O} 72$ line at $18.97 \AA / 653.6 \mathrm{eV}$ and the lower bar to the C52 line at $33.74 \AA / 367.6 \mathrm{eV}$. A displacement to the right (where wavelength increases) corresponds to a redshift.

Figure 8a shows an extended emission region which is elongated mainly along the cross dispersion direction. This alignment, however, is not precise: there are indications for some tilt of the upper and lower wings to the right. As this figure is composed of individual images obtained with a slitless spectrograph, there are generally two possibilities, spectrally or morphologically, for interpreting this tilt:

- In the spectral interpretation, this would be evidence for a redshift. The redshift could be explained by the Doppler effect, as the excited solar wind ions are moving away from us. We should expect to observe a Doppler redshift of

$$
\frac{\delta \lambda}{\lambda} \sim \frac{v_{\mathrm{i}}}{c} \cos (\varphi)
$$

where $v_{\mathrm{i}} \sim 400-800 \mathrm{~km} \mathrm{~s}^{-1}$ is the velocity of the solar wind ions and $\varphi=41.2$ is the phase angle of Mars during the XMM-Newton observation ${ }^{4}$. The Doppler shift of the $15-35 \AA$ emission lines may reach the values of 0.015-0.035 $\AA$ for the slow solar wind and 0.03-0.07 $\AA$ for the fast solar wind. The fact that the observed redshifts decrease with decreasing distance from Mars (along the cross dispersion direction) would be evidence for the fact that the deceleration increases towards Mars. I.e., with increasing density of the Martian exosphere. The deceleration would be a direct consequence of momentum exchange as a byproduct of the charge exchange interactions with atoms in the Martian exosphere.

\footnotetext{
4 Here we ignore the relative velocity between Mars and XMMNewton, which is less than $14.2 \mathrm{~km} \mathrm{~s}^{-1}$ and thus more than one order of magnitude lower.
} 

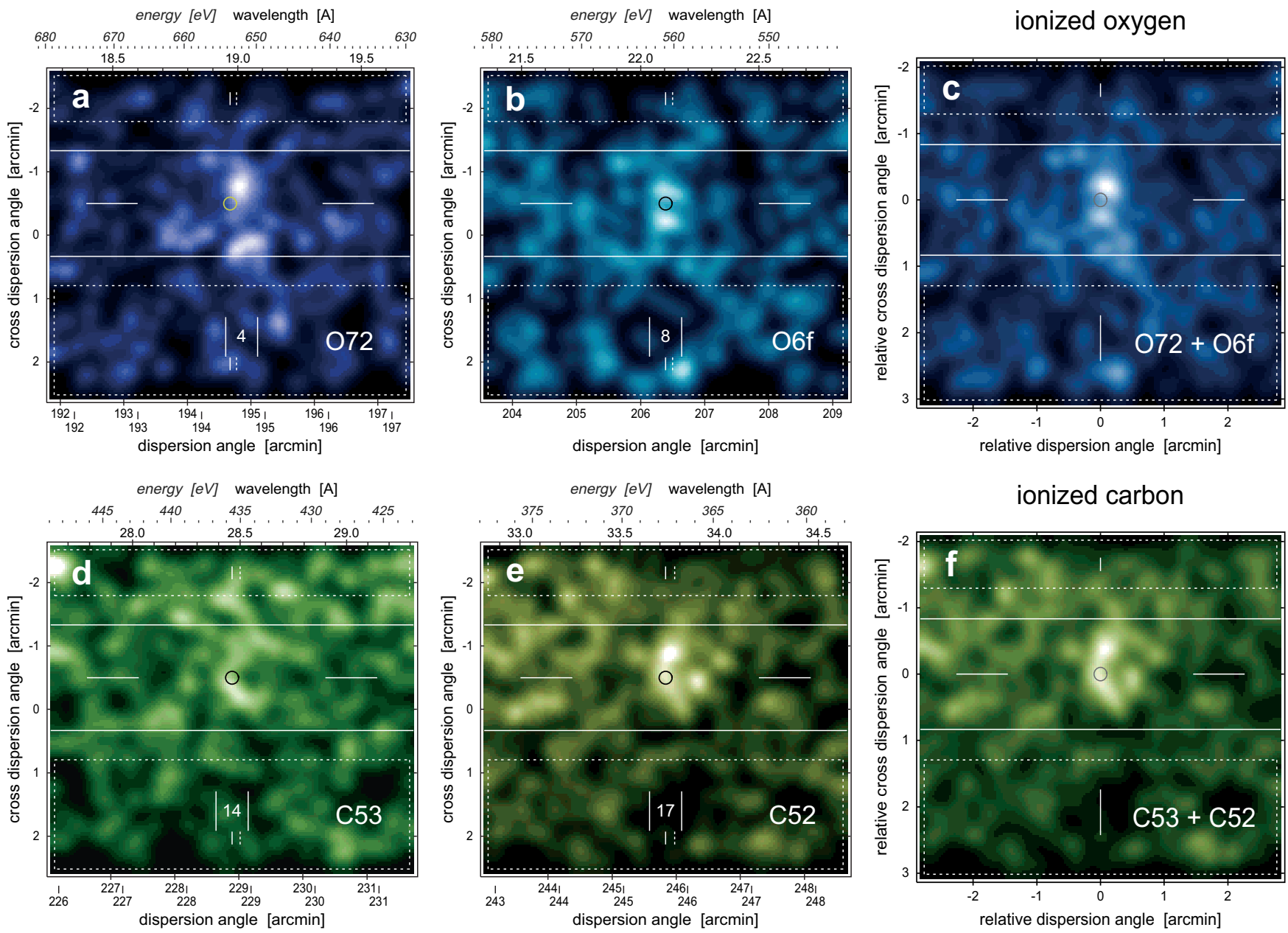

ionized carbon
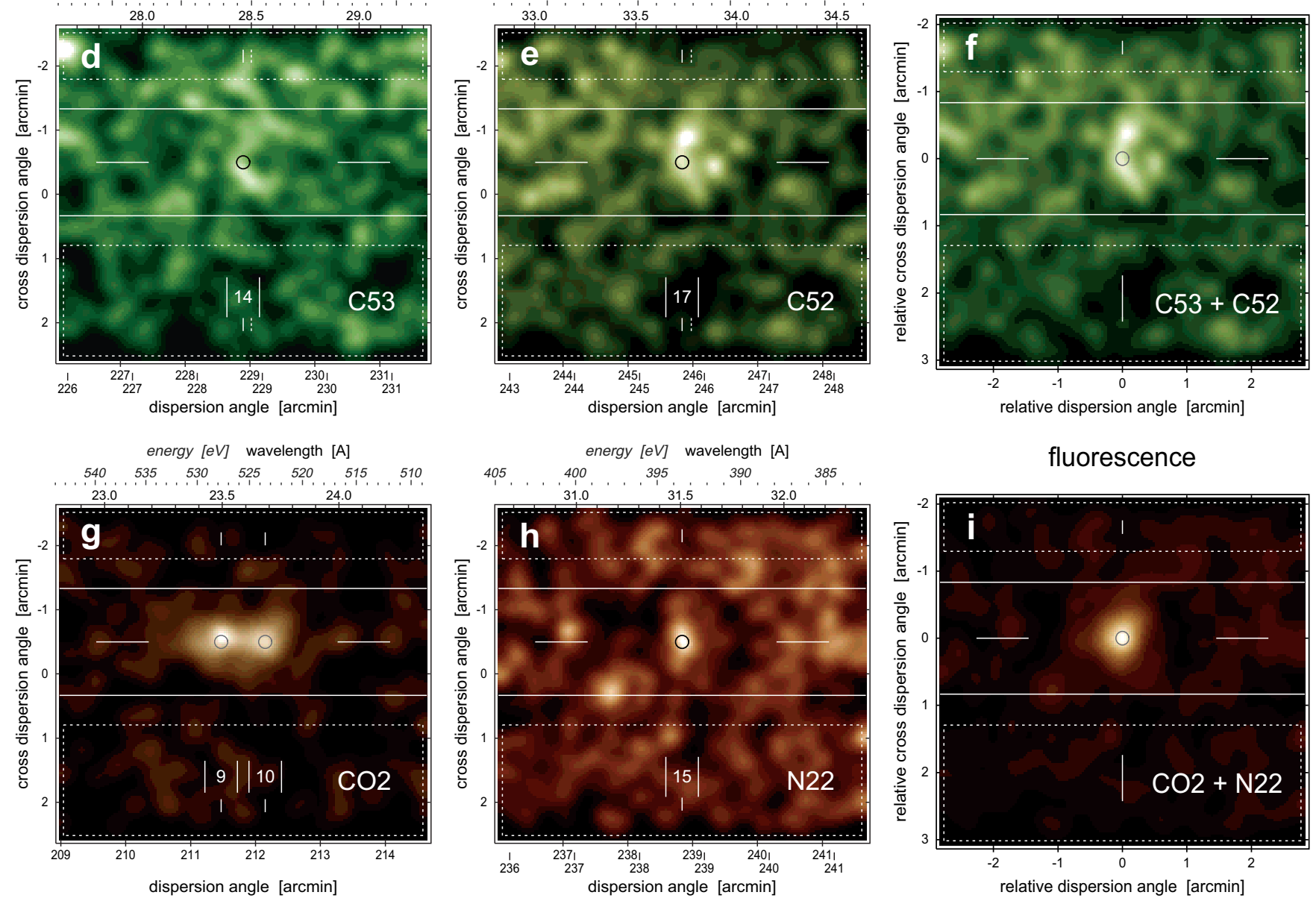

fluorescence



Fig. 7. RGS images of Mars and its halo in the individual emission lines of ionized oxygen (top row), ionized carbon (middle row), and fluorescence of $\mathrm{CO}_{2}$ and $\mathrm{N}_{2}$ molecules (bottom row). The images were corrected for exposure variations, were binned into $2^{\prime \prime} \times 2^{\prime \prime}$ pixels and smoothed with a Gaussian function with $\sigma=8^{\prime \prime} \times 8^{\prime \prime}$. All are displayed at the same angular scale; the dynamic scale, however, was individually adjusted. Data from RGS 1 and RGS 2 were combined whenever available; the upper dispersion angle scale refers to RGS 1 and the lower one (if present) to RGS 2. Wavelength and energy scales are given above each image. The images in the rightmost column are the sum of the images to their left. For the wavelengths/energies of the emission lines, the values in Table $2 b$ were used. These are indicated by the short vertical lines and the circle in the middle, which illustrates the size and expected position of Mars at these emission lines. Dashed vertical lines in frames a, b, d, e indicate the apparent shift along dispersion direction for a redshift of $400 \mathrm{~km} \mathrm{~s}^{-1}$. The solid rectangle refers to the $|y| \leq 50^{\prime \prime}$ region which was used for the spectrum in Fig. 2 and Table 2a, while the dashed rectangles above and below enclose the regions which were used for the background determination. The projected direction to the Sun is towards the left (cf. Fig. 8a). 

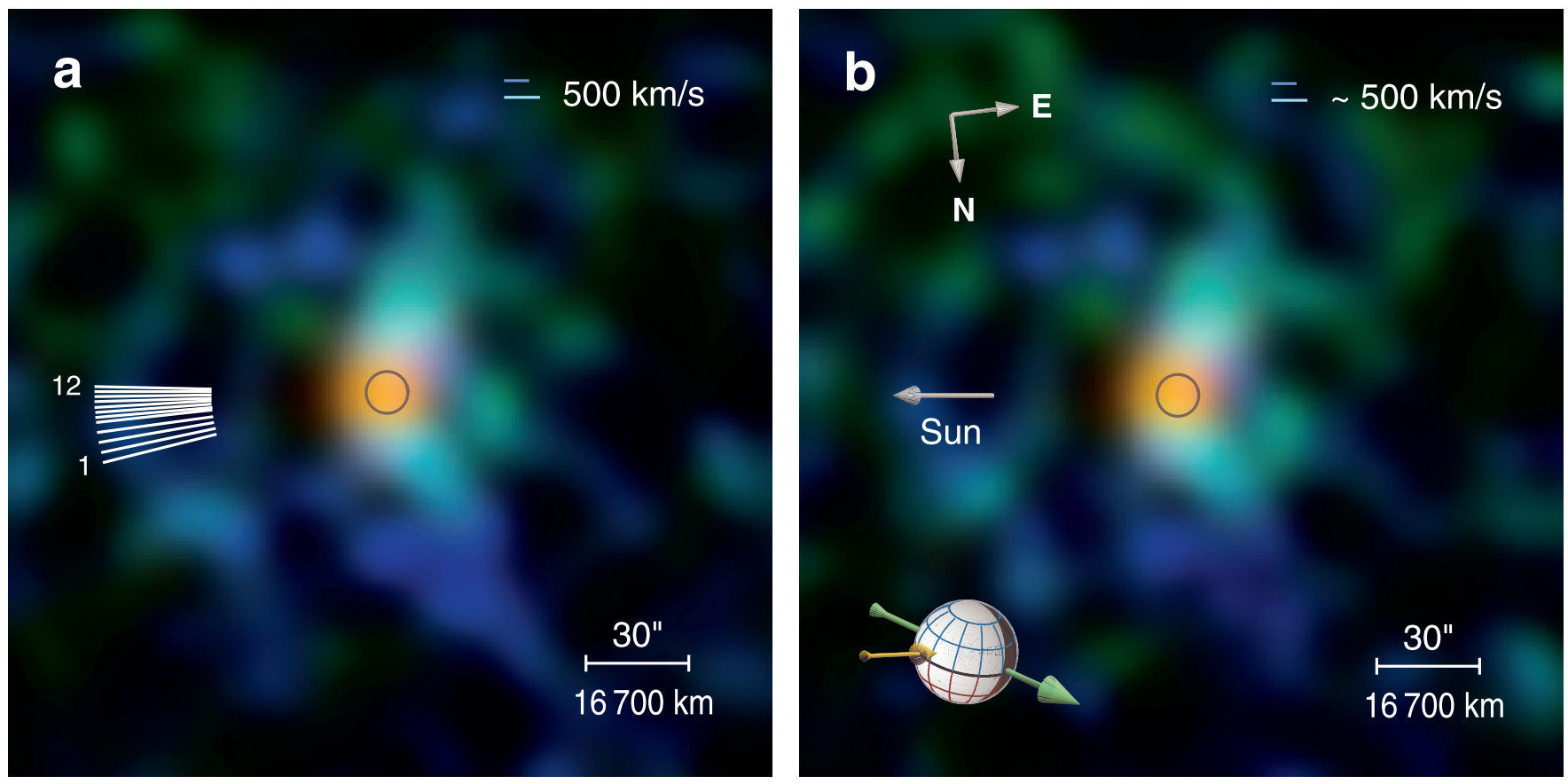

Fig. 8. a) Superposition of the RGS images in Fig. 7, each centered on the wavelength/energy of an individual emission line, with ionized oxygen coded in blue, ionized carbon coded in green, and fluorescence coded in yellow and red. The circle indicates the position and size of Mars; the projected direction of the Sun is towards the left. As the roll angle of the satellite was adjusted for each of the 12 individual pointings in order to minimize the motion of Mars along cross dispersion direction, the position of the Sun with respect to dispersion direction was changing monotonically. Solar directions are labeled for the first and last pointing (cf. Table 1). b) Same as a), but after applying an additional transformation individually to all photons (rotation around the circle at center) so that the projected direction to the Sun is in all cases exactly at left (horizontal arrow). The direction of increasing right ascension ("E") and declination ("N") are given at upper left. The sphere at lower left provides details about the observing geometry: the grid shows areographic coordinates, with blue lines for the southern hemisphere (top) and red lines for the northern hemisphere (bottom). The bright part of the sphere is the sunlit side of Mars. A green arrow indicates its direction of motion, as seen from a stationary point at the position of the Earth. The yellow arrow illustrates the velocity of solar wind particles, emitted radially from the Sun with $400 \mathrm{~km} \mathrm{~s}^{-1}$ with respect to Mars.

- In the morphological interpretation (cf. Fig. 8b), we see an emission region which is most prominent above the poles, but somewhat tilted away from the Sun. This general appearance may be a consequence of the phase angle at which Mars was observed: if the X-ray emission originates preferentially at the sunward side of the Martian halo, then we should see a crescent-like structure when observing it at a phase angle of 41.2. This was the result of numerical simulations (Holmström et al. 2001, Plate 1), which predicted a crescent-like structure that resembles (on a smaller scale) the observed emission in ionized carbon (Fig. 7f). The fact that (i) the emission of ionized carbon extends far above the poles and that (ii) the emission of ionized oxygen emission is observed to occur almost exclusively above the poles could be understood as evidence for an asymmetric density structure in the Martian exosphere, which would be much denser above the poles and towards the night side than towards the Sun.

A straightforward distinction between these two cases would be possible by comparing the negative and positive spectral orders: if the tilt was a spectral effect, then the images at negative and positive spectral orders would be mirrored, while in the case of a morphological effect, the tilt would be oriented in the same way in both orders. Unfortunately, this method cannot be applied here, because RGS works only at negative orders and because both RGS 1 and RGS 2 are oriented in the same way.

The only possibility to distinguish, on a purely observational basis, between these two cases is to utilize the variable direction to the Sun caused by changes of the satellite roll angle (Table 1). The lines left of Mars in Fig. 8a illustrate this direction for the 12 pointings. In Fig. 9, we show the O6f image accumulated during the first three pointings, where the solar direction exhibited the largest deviation from the cross dispersion direction. Despite the low number of photons, there is evidence for a tilt against the cross dispersion direction, approximately the amount expected for an emission morphology directed along the Sun-Mars vector. This could be considered as evidence in favor of the morphological interpretation.

Under the assumption that the cause of the tilt of the emission region is mainly morphological, an improved, sharper image can be obtained by applying to each photon an additional rotation around the center of Mars according to the instantaneous orientation of the detector with respect to the Sun. Figure $8 \mathrm{~b}$ takes this additional rotation into account. A comparison between Figs. $8 \mathrm{a}$ and $8 \mathrm{~b}$ shows that the differences between both images are subtle. This demonstrates that there is little dependence on the assumptions made. It is well possible that we see a superposition of both effects. 


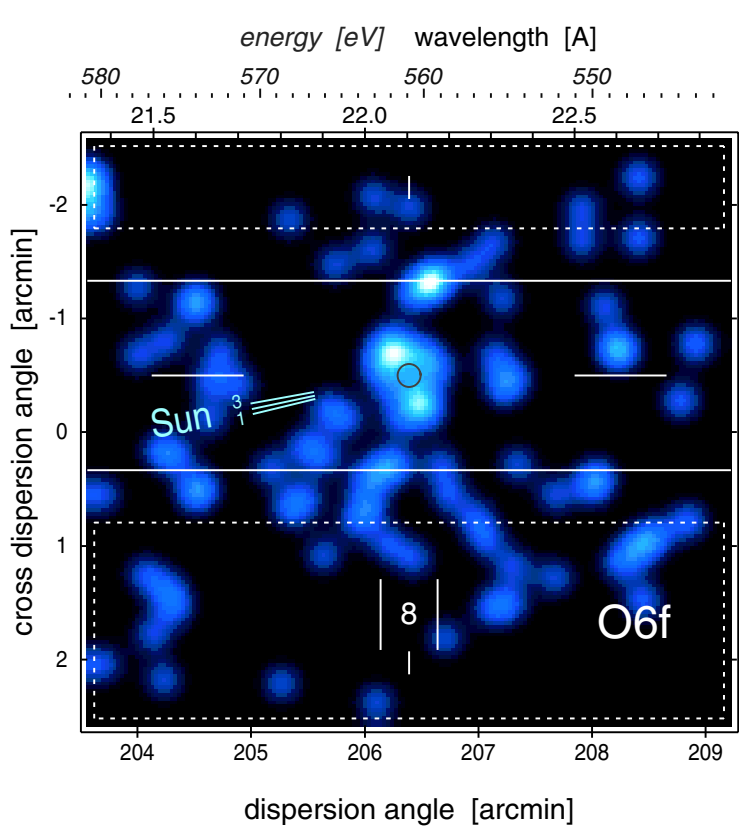

Fig. 9. Same as Fig. 7b, but accumulated only during the pointings 1-3 (Table 1). The direction to the Sun is indicated for these pointings.

\subsection{X-ray fluorescence}

An interesting result of this XMM-Newton Mars observation is that the X-ray fluorescence emission of oxygen consists of two components of similar flux, one at $(523.3 \pm 0.3) \mathrm{eV}$ and the other one at $(528.1 \pm 0.4) \mathrm{eV}$ (Fig. 5 and Tables $2 \mathrm{a}$ and $2 \mathrm{c})$.

This is probably the first astronomical measurement of fine structure in the $\mathrm{X}$-ray fluorescence spectrum of oxygen which is embedded in a $\mathrm{CO}$ or $\mathrm{CO}_{2}$ molecule. For Mars, all previous X-ray data (Dennerl 2002) were obtained with Chandra by direct imaging onto ACIS-I, which cannot resolve this emission feature. For Venus, the only other planet with a dense $\mathrm{CO}_{2}$ atmosphere, X-ray data with high spectral resolution were obtained with Chandra LETG/ ACIS-S (Dennerl et al. 2002). However, due to the combination of low photon statistics, lower dispersion ( $\sim 0.3^{\prime} / \AA$ of LETG compared to $\sim 4^{\prime} / \AA$ for RGS), and the larger angular size of the planet at the time of the observation (22'. 8 diameter of Venus compared to $122^{\prime \prime} 2$ of Mars), the effective spectral resolution was not sufficient for detecting any similar fine structure.

A straightforward explanation for the fact that we are observing two peaks instead of one is that in the $\mathrm{CO}_{2}$ atmosphere of Mars the oxygen atom is embedded in a molecule, where additional electron energy states are available. Laboratory X-ray spectroscopy of $\mathrm{CO}_{2}$ (Nordgren et al. 1997; Gunnelin et al. 1998) shows the presence of the same two lines in the nonresonant X-ray emission spectrum as we observe from Mars. These lines originate from transitions within the four outermost valence orbitals: the line at $528 \mathrm{eV}(\mathrm{CO} 2 \mathrm{a})$ is caused by an electron transition from the $1 \pi_{g}$ orbital (which is almost a pure $2 \mathrm{p}$ orbital around the oxygen atom, and thus similar to the isolated oxygen atom state) into the ground state, while the line at $523 \mathrm{eV}(\mathrm{CO} 2 \mathrm{~b})$ is a superposition of transitions from three orbitals, $4 \sigma_{g}, 3 \sigma_{u}$, and $1 \pi_{u}$, into the ground state.
The laboratory measurements show that the excitation of $\mathrm{CO}_{2}$ with X-ray photons with an energy above the ionization threshold of $540.8 \mathrm{eV}$ leads to isotropic emission with an intensity ratio $I(\mathrm{CO} 2 \mathrm{a}) / I(\mathrm{CO} 2 \mathrm{~b})=1.4 \pm 0.2($ measured at $549.2 \mathrm{eV}$ and $559.2 \mathrm{eV}$; Gunnelin et al. 1998). The corresponding intensity ratio which we obtain from the XMM-Newton Mars observation is $0.8 \pm 0.2$ at $|y| \leq 50^{\prime \prime}$ (Table 2a) and $1.1 \pm 0.3$ at $|y| \leq 10^{\prime \prime}$ (Table 2c).

The ACIS-I spectrum of Mars in the previous Chandra observation was dominated by a single narrow emission line, which appeared at an energy of $(0.65 \pm 0.01) \mathrm{keV}$. This line was interpreted as the oxygen fluorescence line, shifted by $\sim 0.12 \mathrm{keV}$ towards higher energies due to optical loading (Dennerl 2002). The present RGS spectra now clearly show that this interpretation was correct.

In addition to oxygen fluorescence, we also observe fluorescence of nitrogen, at $13 \pm 6 \%$ of the $\mathrm{CO}_{2}$ flux level (Fig. 3 and Table 2c). The X-ray image (Fig. 7h) shows that this emission originates close to Mars, as expected for fluorescence. This line results most likely from the $3 \sigma_{g} \rightarrow 1$ s transition of $\mathrm{N}_{2}$, according to laboratory measurements (Nordgren et al. 1997, Fig. 2). For completeness we note that the carbon fluorescence lines of $\mathrm{CO}_{2}$, which were already observed at Venus (Dennerl et al. 2002) and should also be present at Mars, are just outside the RGS spectral bandpass.

\subsection{The He-like $\mathrm{O}^{6+}$ multiplet}

Among the many emission lines in the RGS spectrum of Mars which are caused by de-excitation of ions, those emitted from $\mathrm{O}^{6+}$ are of particular importance because of their high diagnostic value. These lines are the result of electron transitions between the $n=2$ shell and the $n=1$ ground state shell. As $\mathrm{O}^{6+}$ contains two electrons, there are two possible states of this ion, depending on the relative spin orientation of the electrons: singlet states (mainly $1 \mathrm{~s} 2 \mathrm{~s}{ }^{1} \mathrm{~S}_{0}$ and $1 \mathrm{~s} 2 \mathrm{p}^{1} \mathrm{P}_{1}$ ) and triplet states (mainly $1 \mathrm{~s} 2 \mathrm{~s}{ }^{3} \mathrm{~S}_{1}$ and $1 \mathrm{~s} 2 \mathrm{p}{ }^{3} \mathrm{P}_{0,1,2}$ ). Transitions from triplet states to the ground state require spin changes of the electrons.

While the (spin-forbidden) de-excitation from the singlet state $1 \mathrm{~s} 2 \mathrm{~s}{ }^{1} \mathrm{~S}_{0}$ creates a two photon continuum, the transitions from the other states lead to the emission of discrete lines. Deexcitation from the other singlet state ${ }^{1} \mathrm{P}_{1}$ occurs directly via a (spin-allowed) electric dipole E1 transition, with a decay rate of $3.302 \times 10^{12} \mathrm{~s}^{-1}$ (Savukov et al. 2003). The corresponding emission is usually referred to as resonance line (O6r here).

The transition from the ${ }^{3} \mathrm{~S}_{1}$ triplet state involves a magnetic dipole transition $M 1$ and is a slow process, with a decay rate of $1.047 \times 10^{3} \mathrm{~s}^{-1}$ (Savukov et al. 2003). This transition is usually called forbidden. Although several other forbidden transitions occur in $\mathrm{O}^{6+}$, we follow the general practice and adopt this terminology here (O6f).

Transition from the ${ }^{3} \mathrm{P}_{1}$ triplet state involves an electric dipole E1 transition and is relatively fast, with a decay rate of $5.357 \times 10^{8} \mathrm{~s}^{-1}$ (Savukov et al. 2003). The corresponding line is usually called the intercombination line (O6i). Transition from the ${ }^{3} \mathrm{P}_{2}$ triplet state requires a magnetic quadrupole transition $M 2$, with a decay rate of $3.311 \times 10^{5} \mathrm{~s}^{-1}$ 
(Savukov et al. 2003), and leads to an additional weak intercombination line, at almost the same energy as the ${ }^{3} \mathrm{P}_{1}$ decay. The ${ }^{3} \mathrm{P}_{0}$ triplet state does not decay directly into the ground state, but decays first into the metastable ${ }^{3} S_{1}$ state (see above).

This metastable ${ }^{3} \mathrm{~S}_{1}$ state is diagnostically quite valuable because it has a low radiative transition rate and can be easily de-populated by collisions before radiative decay takes place. Collisional excitation would depopulate first the ${ }^{3} \mathrm{~S}_{1}$ level to the ${ }^{3} \mathrm{P}_{0,1,2}$ level (e.g. Porquet 2000). Thus, the intensity of O6f would decrease and that of O6i would increase. As we do not observe any significant flux from O6i, but from O6f, we can exclude collisional excitation (around Mars) as the origin of the ionization.

The ratio $G=(\mathrm{O} 6 \mathrm{f}+\mathrm{O} 6 \mathrm{i}) / \mathrm{O} 6 \mathrm{r}$ of triplet to singlet transitions is usually less than one for hot plasmas (e.g. Smith et al. 2001). However, if the emission lines result from electron capture by multi-charged ions colliding with neutral gas at low density, the situation is completely different. In this case, $G$ is predicted to be in excess of three (Kharchenko et al. 2003). The value of the $G$ ratio for the $\mathrm{O}^{6+}$ emission induced in the interaction between the solar wind ions and heliospheric hydrogen gas has been evaluated as 6.7 (Pepino et al. 2004), and for the cometary X-rays as 5.8 (Kharchenko 2005). The value which we derive from the Mars spectra is $G \sim 6$ for $|y| \leq 50^{\prime \prime}$ and $G \sim 5$ for $15^{\prime \prime}<|y| \leq 50^{\prime \prime}$. These values, however, are highly uncertain, because only the O6f line is statistically significant (Table 2c). Interestingly, in the disk spectrum (at $|y| \leq 10^{\prime \prime}$ ), the O6r line is clearly present and even brighter than the O6f line (Fig. 3), reducing the ratio to $G=(0.8 \pm 0.6)$.

\subsection{The $\mathrm{O}^{7+} / \mathrm{O}^{6+}$ flux ratio}

This ratio contains information about the abundance of heavy solar wind ions. For the ions $\left[\mathrm{O}^{8+}\right]$ and $\left[\mathrm{O}^{7+}\right]$, an abundance ratio of 0.35 is suggested as typical for the slow solar wind (e.g. Schwadron \& Cravens 2000). A value of $(0.34 \pm 0.07)$ was deduced by Kharchenko et al. (2003) from the Chandra spectrum of comet McNaught-Hartley (C/1999 T1). XMM-Newton observations of the geocoronal and heliospheric X-ray background (Snowden et al. 2004), obtained during the passage of a strong solar wind enhancement, yielded a significantly increased abundance ratio of $0.57 \pm 0.07$.

From the Mars spectrum at $|y| \leq 50^{\prime \prime}$ (Table 2c), we obtain for the ratio of the $\mathrm{O}^{7+}(=\mathrm{O} 74+\mathrm{O} 72)$ flux to the $\mathrm{O}^{6+}$ $(=\mathrm{O} 65+\mathrm{O} 6 \mathrm{r}+\mathrm{O} 6 \mathrm{i}+\mathrm{O} 6 \mathrm{f})$ flux a value of $0.56 \pm 0.16$. If we scale this flux ratio to an ion abundance ratio by assuming that the cross section of $\mathrm{O}^{8+}$ is on average 1.66 higher than that of $\mathrm{O}^{7+}$ (following Snowden et al. 2004), we get $\left[\mathrm{O}^{8+}\right] /\left[\mathrm{O}^{7+}\right]=0.34 \pm 0.10$, which agrees well with the typical slow solar wind value of 0.35 .

\subsection{Luminosity of the disk and halo}

The photon fluxes in Table $2 \mathrm{c}$ can be converted into luminosities, if the angular distribution of the emitted photons is known. As the exosphere of Mars is optically thin to X-ray photons, we can assume isotropic emission there. The luminosity of the

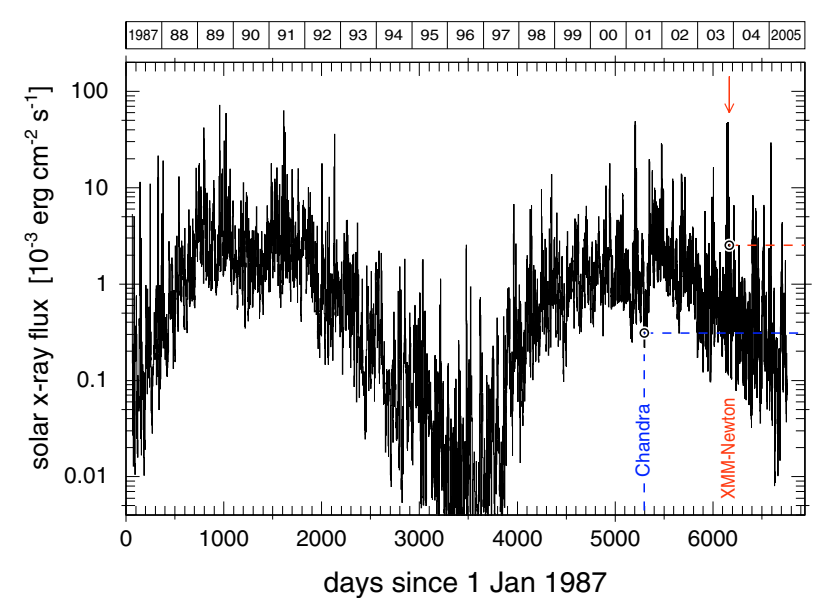

Fig. 10. $1-8 \AA(1.55-12.4 \mathrm{keV})$ solar X-ray flux at $1.0 \mathrm{AU}$, measured with the GOES satellites. The times of the Chandra and XMMNewton observations are marked, together with the corresponding mean solar X-ray flux, which was highly variable during the XMMNewton observation.

X-ray halo $L_{\mathrm{x}}(h)$ results then directly from the photon fluxes $p h\left(i_{h}\right)$ of the halo emission lines observed at an energy $E\left(i_{h}\right)$ :

$L_{\mathrm{x}}(h)=4 \pi \Delta^{2} \sum_{i_{h}} p h\left(i_{h}\right) E\left(i_{h}\right)$

where $\Delta=0.77 \mathrm{AU}$ is the geocentric distance of Mars during the observation. We obtain $L_{\mathrm{x}}(h)=(12.8 \pm 1.4) \mathrm{MW}$ in the spectral band $14-34 \AA$ or $365-880 \mathrm{eV}$ (omitting the N63 line, where the flux determination is highly uncertain).

For the fluorescence radiation, the situation is different, because the atmosphere of Mars (and the planet itself) is optically thick to soft X-rays. In this case, the luminosity can be computed by spherically integrating the flux $f_{\mathrm{x}}\left(\varphi, i_{d}\right)$, observed at the phase angle $\varphi$ in the emission line $i_{d}$, over phase angle:

$L_{\mathrm{X}}(d)=2 \pi \Delta^{2} \int_{0}^{\pi}\left[\sum_{i_{d}} f_{\mathrm{x}}\left(\varphi, i_{d}\right)\right] \sin \varphi \mathrm{d} \varphi$.

The flux $f_{\mathrm{x}}\left(\varphi, i_{d}\right)$ can be obtained by modeling the individual scattering and absorption processes, as described in Dennerl et al. (2002). We obtain $L_{\mathrm{x}}(d)=(3.4 \pm 0.4) \mathrm{MW}$ for fluorescence of oxygen (lines $\mathrm{CO} 2 \mathrm{a}$ and $\mathrm{CO} 2 \mathrm{~b}$ ) and nitrogen (line $\mathrm{N} 22)^{5}$. It is well possible that the disk spectrum contains also some contribution from elastic scattering of solar X-rays, in addition to fluorescence. This contribution, however, should be small. Cravens \& Maurellis (2001) have investigated both processes and found that the X-ray intensity due to fluorescence of $\mathrm{O}$ and $\mathrm{N}$ alone exceeds that of elastic scattering in the broad spectral band $2-120 \AA(0.1-6.2 \mathrm{keV})$ by a factor of 2.4 , and we do not find significant unambiguous evidence for elastic scattering in the RGS spectrum. Therefore, we restrict the calculation of the disk luminosity $L_{\mathrm{x}}(d)$ to the oxygen and nitrogen fluorescence lines in the RGS bandpass.

This luminosity is significantly higher than the $(1.4 \pm 0.2) \mathrm{MW}$ derived from the previous Chandra observation (Dennerl 2002) for oxygen and nitrogen (the carbon

\footnotetext{
5 These values result from the $|y|<50^{\prime \prime}$ spectrum. From the $|y|<$ $10^{\prime \prime}$ spectrum, we get $L_{\mathrm{x}}(d)=(3.7 \pm 0.5) \mathrm{MW}$.
} 
fluorescence is just outside the RGS spectral band). An obvious explanation is found in Fig. 10: although the XMM-Newton observation took place during the declining part of the solar cycle, this observation happened to fall into a period of extreme solar activity, where the mean solar $1-8 \AA$ flux was almost one order of magnitude higher than during the Chandra observation.

The observed luminosity of the X-ray halo is more than one order of magnitude higher than in the previous Chandra observation of Mars, which yielded $L_{\mathrm{x}}(h)=(0.5 \pm 0.2) \mathrm{MW}$ in the energy range $0.5-1.2 \mathrm{keV}$ (Dennerl 2002). The lower value is in good agreement with theoretical predictions (Krasnopolsky 2000; Holmström et al. 2001; Krasnopolsky \& Gladstone 2005).

One reason for the much higher halo luminosity during the XMM-Newton observation is the fact that we observe a considerable flux at large distances from Mars: the Chandra flux was derived from within 3 Mars radii around its center, or $r=30^{\prime \prime}$ at the time of the Chandra observation. Outside this radius, no significant excess of surface brightness relative to the Chandra ACIS-I background level was detectable. During the XMM-Newton observation, this radius would correspond to only $18^{\prime \prime}$, due to the larger geocentric distance of Mars. Thus, the XMM-Newton "halo region", which we have defined here by cross dispersion distances $15^{\prime \prime}<|y| \leq 50^{\prime \prime}$, is almost completely outside the Chandra halo region, and this region contains most of the observed flux (cf. Fig. 8): according to Table $2 \mathrm{c}$, the $\mathrm{X}$-ray luminosity in this region alone is $L_{\mathrm{x}}(h)=(10.0 \pm 1.2) \mathrm{MW}$.

Another reason for the high X-ray luminosity of the halo is the unusual solar activity during the XMM-Newton observation, which produced a highly disturbed solar wind environment around Mars. This is directly confirmed by the XMM-Newton EPIC data (Dennerl et al., in preparation). Unfortunately, Mars Express had not arrived yet at Mars in November 2003, so that no simultaneous in-situ plasma measurements exist.

It is remarkable that the XMM-Newton RGS has the capability to trace the exospheric X-ray emission out to $\sim 8$ Mars radii $(\sim 27000 \mathrm{~km})$, proceeding into exospheric regions far beyond those that have been observationally explored to date. This is particularly interesting because the X-ray emission results directly from charge exchange interactions between atmospheric constituents and solar wind ions, a process which is considered as an important nonthermal escape mechanism and which may be responsible for a significant loss of the Martian atmosphere (Shizgal \& Arkos 1996). Despite this importance, our observational knowledge of the Martian exosphere is still poor and is mainly based on detailed simulations (e.g., Chen \& Cloutier 2003). Thus, X-ray observations, providing a novel method for studying exospheric processes on a global scale, may lead to a better understanding of the present state of the Martian atmosphere and its evolution.

\subsection{Link to cometary $X$-ray emission and the $X$-ray background}

The finding that the X-ray emission of the Martian exosphere is caused by charge exchange interactions between highly charged solar wind heavy ions and neutral gas provides a direct link to cometary X-ray emission (e.g. Lisse et al. 1996; Dennerl et al. 1997; Mumma et al. 1997), which is now understood as the result of the same process (Cravens 1997; Lisse et al. 2001). In fact, the Chandra and XMM-Newton X-ray spectra of comets published so far (Lisse et al. 2001; Krasnopolsky et al. 2002; Dennerl et al. 2003) exhibit many similarities with the X-ray spectrum of the Martian halo.

These comet spectra were taken by direct imaging onto $\mathrm{X}$-ray CCDs, deriving the spectral information from the measured pulse height distribution. This technique yields a spectral resolution of $\sim 10(F W H M \gtrsim 50 \mathrm{eV})$ at $500 \mathrm{eV}$, which is sufficient for detecting bright spectral features, but not sufficient for resolving spectral blends, so that the spectral reconstruction is not always unique. In this respect, RGS spectra of Mars may help in interpreting cometary X-ray spectra: RGS yields a spectral resolution of $\sim 400(F W H M \sim 1.2 \mathrm{eV})$ at $500 \mathrm{eV}$. Unlike the case of highly extended comets in current slitless spectrometers, the X-ray halo around Mars is sufficiently compact that it does not degrade the spectral resolution of RGS to more than $\sim 4 \mathrm{eV}$. Thus, Mars offers a unique opportunity for studying the elusive charge exchange spectra in unprecedented quality.

Such investigations are of general importance to X-ray astronomy, because, triggered by the discovery of cometary $\mathrm{X}$-ray emission, one component of the soft diffuse X-ray background was suspected to be caused by similar processes of solar wind charge exchange in the exosphere of Earth (e.g. Dennerl et al. 1997; Cox 1998; Freyberg 1998) and in the heliosphere (Cravens 2000; Cravens et al. 2001). In fact, recent observations by XMM-Newton (Snowden et al. 2004) and Chandra (Wargelin et al. 2004) have shown the presence of charge exchange induced emission lines in the diffuse X-ray background.

The highest spectral resolution data of the X-ray background have so far been obtained with microcalorimeters flown on a sounding rocket, reaching a $F W H M \sim 9 \mathrm{eV}$ (McCammon et al. 2002). However, this resolution is still inferior to the RGS resolution obtained at the Martian exosphere, and achieving a spectral resolution of $F W H M<5 \mathrm{eV}$ will even be a challenge for future microcalorimeters.

\section{Summary and conclusions}

The XMM-Newton observation of Mars presented here has provided the first unambiguous detection of charge exchange induced X-ray emission from the exosphere of another planet. Based on the RGS data alone, it is already possible to derive a considerable amount of spectral and morphological information about the origin of the Martian X-ray halo. The He-like $\mathrm{O}^{6+}$ multiplet is resolved and dominated by the $2{ }^{3} \mathrm{~S}_{1} \rightarrow 1{ }^{1} \mathrm{~S}_{0}$ transition. This is direct proof that the halo emission is caused by charge exchange processes. Spectral images at individual emission lines reveal that the halo emission occurs preferentially above the Martian poles, that its morphology is different 
between individual ions and ionization states, and that its extent is considerable: with RGS, the emission can be traced out to $\sim 8$ Mars radii. Within this region, an X-ray luminosity of $(12.8 \pm 1.4) \mathrm{MW}$ is found. Although the XMM-Newton observation took place during a time of high solar activity, with disturbed solar wind conditions at Mars, the inferred $\left[\mathrm{O}^{8+}\right] /\left[\mathrm{O}^{7+}\right]$ ratio of $0.34 \pm 0.10$ agrees with the value considered as typical for the slow solar wind.

In addition to the halo emission, fluorescent $\mathrm{X}$-ray radiation from the neutral molecules $\mathrm{CO}_{2}$ and $\mathrm{N}_{2}$ is also detected. Due to the high spectral resolution of RGS, the corresponding images are completely detached from the Martian X-ray halo. They clearly show that the fluorescence radiation is closely confined to the planet, as expected for scattering of solar X-rays in the upper Martian atmosphere. RGS reveals fine structure in the $\mathrm{CO}_{2}$ line, which is resolved into two components of similar flux. One component, at $(528.1 \pm 0.4) \mathrm{eV}$ is caused by the $1 \pi_{g} \rightarrow 1 \mathrm{~s}$ transition, while the other one, at $(523.3 \pm 0.3) \mathrm{eV}$, is a superposition of electron transitions from the orbitals $4 \sigma_{g}$, $3 \sigma_{u}$, and $1 \pi_{u}$, into the ground state. For $N_{2}$, only one line is detected, at $(393.9 \pm 0.5) \mathrm{eV}$, containing $13 \pm 6 \%$ of the combined $\mathrm{CO}_{2}$ flux. The combined luminosity of all three lines is $(3.4 \pm 0.4) \mathrm{MW}$.

Although the Martian halo is an extended source, it is sufficiently compact that, due to its high dispersion, RGS can still achieve a spectral resolution of $\gtrsim 120$ at $500 \mathrm{eV}$. This, combined with a spatial resolution of $\sim 5^{\prime \prime}$ (FWHM) and the fact that RGS is a slitless spectrograph of high sensitivity, not only makes it possible to obtain charge exchange spectra with unprecedented spectral resolution, but to get at the same time also images of the morphological structures originating from specific electron transitions in individual ions. This information is likely to contribute to an improved understanding of the physics of charge exchange in general. Furthermore, the excellent capabilities of XMM-Newton RGS for detailed spectroscopic and morphological studies of the interaction between heavy ions in the solar wind and the Martian exosphere open up a completely new possibility of remote global imaging of planetary exospheres.

Acknowledgements. This work is based on observations obtained with XMM-Newton, an ESA science mission with instruments and contributions directly funded by ESA Member States and NASA. In Germany, the XMM-Newton project is supported by the Bundesministerium für Bildung und Forschung/Deutsches Zentrum für Luft- und Raumfahrt (BMBF/DLR), the Max-Planck-Gesellschaft, and the Heidenhain-Stiftung. V.K. has been supported in this work by the NASA grant NNG04GD57G.

\section{References}

Cash, W. 1979, ApJ, 228, 939

Chen, Y., \& Cloutier, P. A. 2003, J. Geophys. Res., 108, 6
Cox, D. P. 1998, Lecture Notes in Physics (Berlin: Springer Verlag), 506, 121

Cravens, T. E. 1997, Geophys. Res. Lett., 24, 105

Cravens, T. E. 2000, Adv. Space Res., 26, 1443

Cravens, T. E., \& Maurellis, A. N. 2001, Geophys. Res. Lett., 28, 3043

Cravens, T. E., Robertson, I. P., \& Snowden, S. L. 2001, J. Geophys. Res., 106, 24883

den Herder, J. W., Brinkman, A. C., Kahn, S. M., et al. 2001, A\&A, 365, L7

Dennerl, K. 2002, A\&A, 394, 1119

Dennerl, K., Englhauser, J., \& Trümper, J. 1997, Science, 277, 1625

Dennerl, K., Burwitz, V., Englhauser, J., Lisse, C., \& Wolk, S. 2002, A\&A, 386, 319

Dennerl, K., Aschenbach, B., Burwitz, V., et al. 2003, in X-Ray and Gamma-Ray Telescopes and Instruments for Astronomy, ed. H. D. T. J. E. Trümper, 4851

Freyberg, M. J. 1998, Lecture Notes in Physics (Berlin: Springer Verlag), 506, 113

Gunell, H., Holmström, M., Kallio, E., Janhunen, P., \& Dennerl, K. 2004, Geophys. Res. Lett., 31, 22801

Gunnelin, K., Glans, P., Skytt, P., Guo, J.-H., \& Nordgren, J. 1998, Phys. Rev. A, 57, 864

Holmström, M., Barabash, S., \& Kallio, E. 2001, Geophys. Res. Lett., 28,1287

Jansen, F., Lumb, D., Altieri, B., et al. 2001, A\&A, 365, L1

Kharchenko, V. 2005, AIP Conf. Proc., 774, 271

Kharchenko, V., Rigazio, M., Dalgarno, A., \& Krasnopolsky, V. A. 2003, ApJ, 585, L73

Krasnopolsky, V. 2000, Icarus, 148, 597

Krasnopolsky, V. A., Christian, D. J., Kharchenko, V., et al. 2002, Icarus, 160, 437

Krasnopolsky, V. A., \& Gladstone, G. R. 2005, Icarus, 176, 395

Lampton, M., Margon, B., \& Bowyer, S. 1976, ApJ, 208, 177

Lisse, C. M., Dennerl, K., Englhauser, J., et al. 1996, Science, 274, 205

Lisse, C. M., Christian, D. J., Dennerl, K., et al. 2001, Science, 292, 1343

McCammon, D., Almy, R., Apodaca, E., et al. 2002, ApJ, 576, 188

Mumma, M. J., Krasnopolsky, V. A., \& Abbott, M. J. 1997, ApJ, 491, L125

Nordgren, J., Glans, P., Gunnelin, K., et al. 1997, Appl. Phys. A, 65, 97

Pepino, R., Kharchenko, V., Dalgarno, A., \& Lallement, R. 2004, ApJ, 617, 1347

Porquet, D., \& Dubau, J. 2000, A\&AS, 143, 495

Savukov, I. M., Johnson, W. R., \& Safronova, U. I. 2003, in Atomic data and Nuclear Data Tables, 85, 83

Schwadron, N. A., \& Cravens, T. E. 2000, ApJ, 544, 558

Shizgal, B. D., \& Arkos, G. G. 1996, Rev. Geoph., 34, 483

Smith, R. K., Brickhouse, N. S., Liedahl, D. A., \& Raymond, J. C. 2001, ApJ, 556, L91

Snowden, S. L., Collier, M. R., \& Kuntz, K. D. 2004, ApJ, 610, 1182

Wargelin, B. J., Markevitch, M., Juda, M., et al. 2004, ApJ, 607, 596 